\title{
Design, synthesis and anticonvulsant activity of new Diacylthiosemicarbazides
}

\author{
O. V. Kholodniak, V. V. Stavytskyi, M. S. Kazunin, N. V. Bukhtiayrova, G. G. Berest, \\ I. F. Belenichev, S. I. Kovalenko \\ Zaporizhia State Medical University \\ 26, Mayakovsky avenue, Zaporizhzhia, Ukraine, 69035 \\ alena.holodniak@gmail.com
}

\begin{abstract}
Aim. A targeted search for anticonvulsant agents among unknown diacylthiosemicarbazides with the analysis of the structure-activity relationship (SAR-analysis). Methods. Organic synthesis; molecular docking; spectral methods; pentylenetetrazole convulsions, statistical methods. Results. A strategy of search for new anticonvulsant agents among unknown diacylthiosemicarbazides has been developed. It included virtual-oriented screening towards [the] active centers of enzymes and sodium channels that underlie the mechanism of antiepileptic drugs activity. The synthesis of diacylthiosemicarbazides was carried out by the in situ method, namely, accomplishing the interaction of cycloalkanecarbonyl chlorides with ammonium isothiocyanate and the subsequent nucleophilic addition of cycloalkyl- (aralkyl-, aryl-, hetaryl-) carboxylic acid hydrazides. The peculiarities of the structure of the synthesized compounds were confirmed by spectral methods (LCMS and ${ }^{1} \mathrm{H}$ NMR spectra). Biological screening showed that diacylthiosemicarbazides (2) in the experimental model of pentyleneterazole seizures in rats increased the latency period of seizures by $2.77-7.82$ times, reduced the duration of tonic-clonic seizures by $1.23-5.59$ minutes and prevented mortality by $30-60 \%$, relative to the control group of animals. It was shown that diacylthiosemicarbazides $(\mathbf{2 . 6}, \mathbf{2 . 1 5}$, 2.22, 2.18) with cyclopropane- or cyclopentanecarboxamide groups show the anticonvulsant activity that exceeds that of the reference drug Depakine or competes with it. Conclusions. A range of new diacylthiosemicarbazides were obtained and the primary screening of their anticonvulsant activity was performed, the SAR-analysis was provided, and the hit-compound was identified for further in-depth pharmacological studies.
\end{abstract}

Keyword s: diacylthiosemicarbazides, design, synthesis, pentylenetetrazole convulsions, anticonvulsant activity.

(C) 2021 O. V. Kholodniak et al.; Published by the Institute of Molecular Biology and Genetics, NAS of Ukraine on behalf of Biopolymers and Cell. This is an Open Access article distributed under the terms of the Creative Commons Attribution License (http://creativecommons.org/licenses/by/4.0/), which permits unrestricted reuse, distribution, and reproduction in any medium, provided the original work is properly cited 


\section{Introduction}

Nowadays, antiepileptic drugs (AEDs) such as hydantoin derivatives (phenytoin, ethosuximide), pyrimidine (phenobarbital, primidone), benzazepines (carbamazepine) and benzodiazepines (diazepam, lorazepam, clonazepam) are widely used. They cannot be considered perfect because of insufficient effectiveness and safety. They have significant side effects and cannot adequately control seizures in some cases [1-5]. However, the drugs of the last generation (valproate, vigabatrin, gabapentin, pregabalin, felbamate, tiagabine, topiramate etc.) show significant improvements in pharmacokinetics and pharmacodynamics compared to the «classic» AEDs. Among them, the most promising are the drugs with small molecules that readily cross the blood-brain barrier (BPH) and inhibit the activity of $\gamma$-aminobutyric acid aminotransferase (GABAAT), a pyridoxal 5'-phosphate (PLP)-dependent enzyme that degrades GABA [6]. At the same time despite a large number of new AEDs, not all of them are able to prevent or delay the onset of epileptic states in a significant number of patients. Additionally, most of them have side effects, namely, weakening cognitive processes, impairing memory and affecting the rapid reproduction of engrams [1, 4]. Obviously, the unwanted side effects and inability to control the main types of epilepsy by AEDs, stimulate the researchers to look for innovative anticonvulsants with more favorable pharmacotherapeutic profile.

The modification products of the carboxyl group of alkyl- and cycloalkylcarboxylic acids (amides, carbamates, semicarbazides, etc.) are one of the promising classes of organic com- pounds in terms of anticonvulsants design [3, 4, 7-14]. Firstly, these structural fragments are present in most molecules of both approved for use and experimental anticonvulsant drugs $[1,4]$. Secondly, such modification has been studied on valproic acid and is promising, in view of the teratogenicity and hepatotoxicity reduction and a positive epileptogenic effect (Fig. 1) [4]. The fact that substituted ureas, semicarbazides and their sulfur-containing analogues are actively studied for anticonvulsant activity is an additional confirmation of the prospects of this approach. Their combination with saturated substituents (cyclopropane, cyclocyclohexane, cycloheptane, citral, carvone, camphor) is a favorable factor for the appearance of biological activity [15-18]. It is important, that additional introduction of fragments with lipophilic properties (lipophilic domain) and donor-acceptor properties (p-charge) to the thiosemicarbazide residue (hydrogen bonding domain) is necessary for the interaction with the active receptor center of the corresponding protein target. Therefore, this modification will show an effect of the «pharmacophore» (cycloalkyl, amide and acylthiosemicarbazide) fragments on the anticonvulsant activity manifestation. Virtual screening of the studied ligands in the active sites $\mathrm{GABA}_{\mathrm{A}}, \mathrm{GABA}_{\mathrm{T}}$ and NavMs will allow the determination of the compounds for researching an experimental model of pentylenetetrazole seizures.

The aim of this research is a virtual targetoriented screening, synthesis and study of diacylthiosemicarbazides for anticonvulsant activity in rats models of pentylenetetrazole sei- 


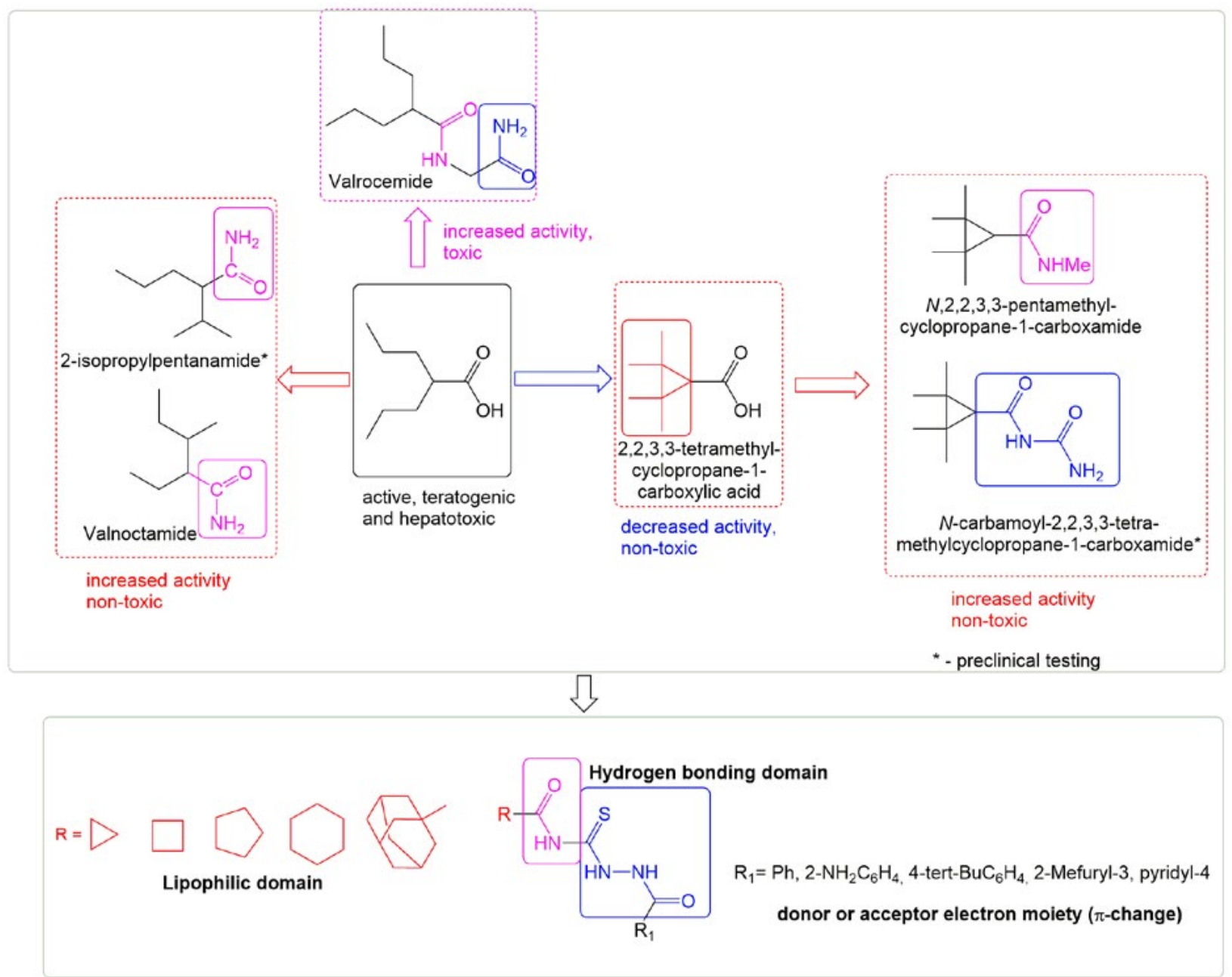

Fig. 1. The methods of modification of valproate and strategies for novel anticonvulsants design among diacylhiosemicarbazides derivatives

zures with SAR-analysis for further directed search for effective drugs.

\section{Materials and Methods}

\section{General Methods}

Melting points were determined in open capillary tubes in a «Mettler Toledo MP 50» apparatus and were uncorrected. The elemental analyses $(\mathrm{C}, \mathrm{H}, \mathrm{N}, \mathrm{S})$ were performed using the ELEMENTAR vario EL cube analyzer (USA). Analyses were indicated by the symbols of the elements or functions within $\pm 0.3 \%$ of the theoretical values. IR spectra (4000$600 \mathrm{~cm}^{-1}$ ) were recorded on a Bruker ALPHA FT-IR spectrometer (Bruker Bioscience, Germany) using a module for measuring attenuated total reflection (ATR). ${ }^{1} \mathrm{H}$ NMR spec- 
tra $(400 \mathrm{MHz})$ were recorded on a VarianMercury 400 spectrometer (Varian Inc., Palo Alto, CA, USA) with TMS as internal standard in DMSO- $d_{6}$ solution. LC-MS were recorded using the chromatography/mass spectrometric system, which consists of high-performance liquid chromatography «Agilent 1100 Series» (Agilent, Palo Alto, CA, USA) equipped with diode-matrix and mass-selective detector «Agilent LC/MSD SL» (atmospheric pressure chemical ionization - APCI). Electron impact mass spectra (EI-MS) were recorded on a Varian $1200 \mathrm{~L}$ instrument at $70 \mathrm{eV}$ (Varian, USA).

Cycloalkanecarbonyl chlorides (1.1-1.5) were synthesized by the known method [19, 20]. Other starting materials and solvents were obtained from commercially available sources and used without additional purification.

The general method synthesis of the $\mathrm{N}-(2-$ alkyl-(aroyl-, hetaroyl-)hydrazine-1-carbonothioyl)cycloalkanecarboxamides (2.1-2.21). To a solution of corresponding $0.01 \mathrm{~mol}$ cycloalkanecarbonyl chlorides (1.1-1.4) in 20 $\mathrm{mL}$ of acetonitrile, $0.76 \mathrm{~g}(0.01 \mathrm{~mol})$ of ammonium isothiocyanate were added and stirred at $80^{\circ} \mathrm{C}$ for $30 \mathrm{~min}$. The mixture was cooled down to r.t. and $0.01 \mathrm{~mol}$ of corresponding hydrazides of carboxylic acids was added and stirred at $80^{\circ} \mathrm{C}$ for $90 \mathrm{~min}$. The solution was cooled down, poured into the water. The formed precipitate was filtrated, dried and recrystallized from methanol. Synthesized compounds (2.2-2.27) were white or light yellow, insoluble in water and ether, soluble in alcohols, dioxane and DMF.

$\mathrm{N}$-(2-(Cyclopropanecarbonyl)hydrazine1-carbonothioyl)cyclopropanecarboxamide (2.1). Yield: $52 \%$; Mp.: $192-193{ }^{\circ} \mathrm{C} ;{ }^{1} \mathrm{H}$ NMR
(400 MHz, DMSO-d 6 ), $\delta: 13.78$ (s, 1H, -C(S) $\mathrm{NH}-$ ), 12.58 (br. s, 1H, -C(O)NH-), 10.77 (br. s, $1 \mathrm{H},-\mathrm{HN}-\mathrm{NH}-\mathrm{C}(\mathrm{O})-), 3.02(\mathrm{~m}, 2 \mathrm{H}$, cyclopropyl $\mathrm{H}-1), 2.06\left(\mathrm{~m}, 2 \mathrm{H}\right.$, cyclopropyl $\mathrm{H}-2_{\mathrm{eq}}$ ), $1.88\left(\mathrm{~m}, 2 \mathrm{H}\right.$, cyclopropyl $\left.3_{\mathrm{eq}}\right), 1.03-0.71(\mathrm{~m}$, $4 \mathrm{H}$, cyclopropyl $\left.2_{\mathrm{ax}}, 3_{\mathrm{ax}}\right)$; LC-MS, $\mathrm{m} / \mathrm{z}=228$ $[\mathrm{M}+1]$; Anal. Calcd. for $\mathrm{C}_{9} \mathrm{H}_{13} \mathrm{~N}_{3} \mathrm{O}_{2} \mathrm{~S}: \mathrm{C}$, 47.56; H, 5.77; N, 18.49; S, 14.11; Found: C, $47.63 ; \mathrm{H}, 5.83 ; \mathrm{N}, 18.56 ; \mathrm{S}, 14.19$.

$N$-(2-(2-Phenoxyacetyl)hydrazine-1-carbonothioyl)cyclopropanecarboxamide (2.2). Yield: $70 \%$; Mp.: 194-195 ${ }^{\circ} \mathrm{C}$; ${ }^{1} \mathrm{H}$ NMR (400 MHz, DMSO-d $\mathrm{d}_{6}$ ), $\delta: 12.62$ (br.s, $1 \mathrm{H}$, -C(S)NH-), 11.77 (s, 1H, -C(O)NH-), 10.73 (br.s. 1H, -HNNHC(O)-), 7.27 (t, J = 7.8 Hz, 2H, Ph H-3,5), 6.99-6.91 (m, 3H, Ph H-2,4,6), $4.65\left(\mathrm{~s}, 2 \mathrm{H},-\mathrm{CH}_{2} \mathrm{OPh}\right), 2.12-2.03(\mathrm{~m}, 1 \mathrm{H}$, cyclopropyl H-1), 1.01-0.88 (m, 4H, cyclopropyl H-2 $\left.2_{\text {eq }}, 2_{\text {ax }}, 3_{\text {eq }}, 3_{\mathrm{ax}}\right) ; \mathrm{LC}-\mathrm{MS}, \mathrm{m} / \mathrm{z}=294$ $[\mathrm{M}+1]$; Anal. Calcd. for $\mathrm{C}_{13} \mathrm{H}_{15} \mathrm{~N}_{3} \mathrm{O}_{3} \mathrm{~S}$ : C, 53.23; H, 5.15; N, 14.32; S, 10.93; Found: C, $53.29 ; \mathrm{H}, 5.21 ; \mathrm{N}, 14.38 ; \mathrm{S}, 11.01$.

N-(2-(2-(Phenylthio)acetyl)hydrazine1-carbonothioyl)cyclopropanecarboxamide (2.3). Yield: 77 \%; Mp.: $187-188^{\circ} \mathrm{C} ;{ }^{1} \mathrm{H}$ NMR (400 MHz, DMSO-d $\mathrm{d}_{6}$ ), $\delta: 12.69$ (br.s, $1 \mathrm{H}$, -C(S)NH-), 11.68 (s, 1H, -C(O)NH-), 10.99 (br.s, 1H, -HNNHC(O)-), 7.40 (d, J = 7.7 Hz, $2 \mathrm{H}, \mathrm{Ph} \mathrm{H}-2,6), 7.28$ (t, J = 7.7 Hz, 2H, Ph $\mathrm{H}-3,5), 7.17$ (t, J = 7.4 Hz, 1H, Ph H-4), 3.76 $\left(\mathrm{s}, 2 \mathrm{H},-\mathrm{CH}_{2} \mathrm{SPh}\right), 2.10-2.01(\mathrm{~m}, 1 \mathrm{H}$, cyclopropyl H-1), 0.99-0.86 (m, 4H, cyclopropyl $\left.\mathrm{H}-2_{\text {eq }}, 2_{\text {ax }}, 3_{\text {eq }}, 3_{\text {ax }}\right) ; \mathrm{LC}-\mathrm{MS}, \mathrm{m} / \mathrm{z}=310[\mathrm{M}+1]$; Anal. Calcd. for $\mathrm{C}_{13} \mathrm{H}_{15} \mathrm{~N}_{3} \mathrm{O}_{2} \mathrm{~S}_{2}: \mathrm{C}, 50.47 ; \mathrm{H}$, 4.89; N, 13.58; S, 20.72; Found: C, 50.53; H, $4.93 ; \mathrm{N}, 14.04 ; \mathrm{S}, 20.78$.

(N-(2-Benzoylhydrazine-1-carbonothioyl) cyclopropanecarboxamide (2.4). Yield: $75 \%$; Mp.: $204-206{ }^{\circ} \mathrm{C} ;{ }^{1} \mathrm{H}$ NMR $(400 \mathrm{MHz}$, 
DMSO-d $\left.{ }_{6}\right), \delta: 12.40(\mathrm{~s}, 1 \mathrm{H},-\mathrm{C}(\mathrm{S}) \mathrm{NH}-), 11.76$ (s, 1H, -C(O)NH-), 10.81 (s, 1H, -HN-NH$\mathrm{C}(\mathrm{O})-), 7.91$ (d, J = 7.6 Hz, 2H, Ph-2,6), 7.57$7.41(\mathrm{~m}, 3 \mathrm{H}, \mathrm{Ph}-3,4,5), 2.16-2.05(\mathrm{~m}, 1 \mathrm{H}$, cyclopropyl H-1), 1.04-0.89 (m, 4H, cyclopropyl H-2 $\left.2_{\text {eq }}, 2_{\mathrm{ax}}, 3_{\mathrm{eq}}, 3_{\mathrm{ax}}\right) ; \mathrm{LC}-\mathrm{MS}, \mathrm{m} / \mathrm{z}=264$ $[\mathrm{M}+1], 265[\mathrm{M}+2]$; Anal. Calcd. for $\mathrm{C}_{12} \mathrm{H}_{13} \mathrm{~N}_{3} \mathrm{O}_{2} \mathrm{~S}$ : C, 54.74; H, 4.98; N, 15.96; S, 12.18; Found: C, 54.79; H, 5.03; N, 16.02; S, 12.24 .

$\mathrm{N}$-(2-(2-Aminobenzoyl)hydrazine-1-carbonothioyl)cyclopropanecarboxamide (2.5). Yield: $81 \%$; Mp.: $186-189{ }^{\circ} \mathrm{C} ;{ }^{1} \mathrm{H}$ NMR $(400 \mathrm{MHz}$, DMSO-d $\left.\mathrm{d}_{6}\right), \delta: 12.52(\mathrm{~s}, 1 \mathrm{H},-\mathrm{C}(\mathrm{S}) \mathrm{NH}-), 11.74$ (s, 1H, -C(O)NH-), 8.27-7.24 (m, 3H, Ar H-6, $\left.\mathrm{NH}_{2}\right), 7.15(\mathrm{t}, \mathrm{J}=7.7 \mathrm{~Hz}, 1 \mathrm{H}, \mathrm{Ph}-4), 6.72$ (d, $\mathrm{J}=8.3 \mathrm{~Hz}, 1 \mathrm{H}, \mathrm{Ph}-3), 6.53(\mathrm{t}, \mathrm{J}=7.5 \mathrm{~Hz}, 1 \mathrm{H}$, $\mathrm{Ph}-5), 2.10$ (p, J = 8.4 Hz, 1H, cyclopropyl H-1), 1.03-0.88 (m, 4H, cyclopropyl H-2 $2_{\text {eq }} 2_{\text {ax }}$, $\left.3_{\mathrm{eq}}, 3_{\mathrm{ax}}\right) ; \mathrm{LC}-\mathrm{MS}, \mathrm{m} / \mathrm{z}=279$ [M+1], $280[\mathrm{M}+2]$; Anal. Calcd. for $\mathrm{C}_{12} \mathrm{H}_{14} \mathrm{~N}_{4} \mathrm{O}_{2} \mathrm{~S}: \mathrm{C}, 51.78 ; \mathrm{H}$, 5.07; N, 20.13; S, 11.52 ; Found: C, 51.82; H, $5.13 ; \mathrm{N}, 20.18 ; \mathrm{S}, 11.59$.

N-(2-Isonicotinoylhydrazine-1carbonothioyl)cyclopropanecarboxamide (2.6). Yield: $72 \%$; Mp.: $182-186{ }^{\circ} \mathrm{C} ;{ }^{1} \mathrm{H}$ NMR (400 MHz, DMSO-d $)_{6}$ ), $\delta: 12.27$ (s, $1 \mathrm{H},-\mathrm{C}(\mathrm{S})$ NH-), 11.80 (s, 1H, -C(O)NH-), 11.20 (s, 1H, -HNNH-C(O)-), 8.70 (d, J = 5.3 Hz, 2H, Pyr $\mathrm{H}-3,5), 7.80(\mathrm{~d}, \mathrm{~J}=5.1 \mathrm{~Hz}, 2 \mathrm{H}$, Pyr H-2,6), 2.15-2.06 (m, 1H, cyclopropyl H-1), 1.03$0.90\left(\mathrm{~m}, 4 \mathrm{H}\right.$, cyclopropyl H-2 $\left.2_{\mathrm{eq}}, 2_{\mathrm{ax}}, 3_{\mathrm{eq}}, 3_{\mathrm{ax}}\right)$; LC-MS, $\mathrm{m} / \mathrm{z}=265[\mathrm{M}+1]$; Anal. Calcd. for $\mathrm{C}_{11} \mathrm{H}_{12} \mathrm{~N}_{4} \mathrm{O}_{2} \mathrm{~S}$ : C, 49.99; H, 4.58; N, 21.20; $\mathrm{S}$, 12.13; Found: C, 50.03; H, 4.60; N, 21.16; S, 12.18 .

$N-(2-(2-P h e n o x y a c e t y l) h y d r a z i n e-1-c a r-$ bonothioyl)cyclobutanecarboxamide (2.7). Yield: $55 \%$; Mp.: $188-193{ }^{\circ} \mathrm{C}$; ${ }^{1} \mathrm{H}$ NMR
(400 MHz, DMSO-d $)_{6}$ ), $\delta: 12.29$ (s, 1H, -C(S) $\mathrm{NH}-), 11.21$ (s, 1H, -C(O)NH-), 10.17 (s, 1H, -HNNHC(O)-), 7.37-7.21 (m, 2H, Ph H-3,5), 7.10-6.90 (m, 3H, Ph H-2,4,6), 5.40 (s, 2H, $\left.-\mathrm{CH}_{2} \mathrm{OPh}\right), 3.38(\mathrm{p}, \mathrm{J}=8.4 \mathrm{~Hz}, 1 \mathrm{H}$, cyclobutyl $\mathrm{H}-1), 2.34-2.17$ (m, 4H, cyclobutyl H-4 eq, $2_{\text {eq }}$, $\left.2_{\mathrm{ax}}, 4_{\mathrm{ax}}\right), 2.07-1.80\left(\mathrm{~m}, 2 \mathrm{H}\right.$, cyclobutyl $\mathrm{H}-3_{\mathrm{eq}}$, $\left.3_{\mathrm{ax}}\right)$; LC-MS, $\mathrm{m} / \mathrm{z}=308$ [M+1]; Anal. Calcd. for $\mathrm{C}_{14} \mathrm{H}_{17} \mathrm{~N}_{3} \mathrm{O}_{3} \mathrm{~S}: \mathrm{C}, 54.71 ; \mathrm{H}, 5.58 ; \mathrm{N}, 13.67$; S, 10.43; Found: C, 54.77; H, 5.63; N, 13.72; $\mathrm{S}, 10.50$.

$\mathrm{N}-(2-(2-($ Phenylthio $)$ acetyl) hydrazine1-carbonothioyl)cyclobutanecarboxamide (2.8). Yield: $72 \%$; Mp.: $130-133{ }^{\circ} \mathrm{C}$; ${ }^{1} \mathrm{H}$ NMR (400 MHz, DMSO-d ${ }_{6}$ ), $\delta: 12.51$ (br. s, $1 \mathrm{H}$, -C(S)NH-), 11.38 (br.s, $1 \mathrm{H},-\mathrm{C}(\mathrm{O}) \mathrm{NH}-), 10.27$ (br.s, 1H, -HNNHC(O)-), 7.40-7.36 (m, 2H, Ph H-3,5), 7.32-7.29 (m, 2H, Ph H-2,6), 7.28$7.19(\mathrm{~m}, 1 \mathrm{H}, \mathrm{Ph} \mathrm{H}-4), 3.84$ (s, $\left.2 \mathrm{H},-\mathrm{CH}_{2} \mathrm{SPh}\right)$, $3.37(\mathrm{~m}, 1 \mathrm{H}$, cyclobutyl H-1), 2.21-2.10 (m, $4 \mathrm{H}$, cyclobutyl H-4 $\left.4_{\text {eq }}, 2_{\text {eq }}, 2_{\text {ax }}, 4_{\text {ax }}\right), 2.03-1.79$ ( $\mathrm{m}, 2 \mathrm{H}$, cyclobutyl $\mathrm{H}-3_{\text {eq }}, 3_{\mathrm{ax}}$ ); LC-MS, $\mathrm{m} / \mathrm{z}=$ $324[\mathrm{M}+1]$; Anal. Calcd. for $\mathrm{C}_{14} \mathrm{H}_{17} \mathrm{~N}_{3} \mathrm{O}_{2} \mathrm{~S}_{2}$ : C, 51.99; H, 5.30; N, 12.99; S, 19.83; Found: C, 52.04; H, 5.36; N, 13.03; S, 19.89 .

N-(2-Benzoylhydrazine-1-carbonothioyl) cyclobutanecarboxamide (2.9). Yield: $57 \%$; Mp.: $163-167{ }^{\circ} \mathrm{C}$; ${ }^{1} \mathrm{H}$ NMR $(400 \mathrm{MHz}$, DMSO-d ${ }_{6}$ ), $\delta: 12.40$ (br.s, $1 \mathrm{H},-\mathrm{C}(\mathrm{S}) \mathrm{NH}-$ ), 11.35 (br.s, 1H, -C(O)NH-), 10.88 (br.s, $1 \mathrm{H}$, -HN-NH-C(O)-), 7.91 (d, J = 7.5 Hz, 2H, Ph2,6), 7.59-7.52 (m, 1H, Ph-4), 7.47 (t, J = $7.4 \mathrm{~Hz}, 2 \mathrm{H}, \mathrm{Ph}-3,5), 3.42$ (p, J = 8.5 Hz, $1 \mathrm{H}$, cyclobutyl H-1), 2.37-2.09 (m, 4H, cyclobutyl $\left.\mathrm{H}-4_{\mathrm{eq}}, 2_{\mathrm{eq}}, 2_{\mathrm{ax}}, 44_{\mathrm{ax}}\right), 2.07-1.81(\mathrm{~m}, 2 \mathrm{H}$, cyclobutyl $\left.\mathrm{H}-3_{\mathrm{eq}}, 3_{\mathrm{ax}}\right) ; \mathrm{LC}-\mathrm{MS}, \mathrm{m} / \mathrm{z}=278[\mathrm{M}+1]$; Anal. Calcd. for $\mathrm{C}_{13} \mathrm{H}_{15} \mathrm{~N}_{3} \mathrm{O}_{2} \mathrm{~S}$ : C, 56.30; $\mathrm{H}$, 5.45; N, 15.15; S, 11.56; Found: C, 56.36; H, $5.51 ; \mathrm{N}, 15.20 ; \mathrm{S}, 11.61$. 
$N$-(2-(2-Aminobenzoyl) hydrazine-1-carbonothioyl)cyclobutanecarboxamide (2.10). Yield: $53 \%$; Mp.: $201-202{ }^{\circ} \mathrm{C} ;{ }^{1} \mathrm{H}$ NMR (400 MHz, DMSO-d 6 ), $\delta: 12.52$ (s, $1 \mathrm{H},-\mathrm{C}(\mathrm{S}) \mathrm{NH}-)$, $11.32(\mathrm{~s}, 1 \mathrm{H},-\mathrm{C}(\mathrm{O}) \mathrm{NH}-), 8.00-7.30(\mathrm{~m}, 3 \mathrm{H}$, Ar H-6, $\mathrm{NH}_{2}$ ), 7.16 (t, J = 7.7 Hz, 1H, Ar H-4), $6.73(\mathrm{~d}, \mathrm{~J}=8.3 \mathrm{~Hz}, 1 \mathrm{H}, \mathrm{Ar} \mathrm{H}-3), 6.54(\mathrm{t}, \mathrm{J}=$ $7.5 \mathrm{~Hz}, 1 \mathrm{H}, \mathrm{Ar} \mathrm{H}-5), 3.41$ (p, J = 8.4 Hz, 1H, cyclobutyl H-1), 2.37-2.09 (m, 4H, cyclobutyl $\left.\mathrm{H}-4_{\text {eq }}, 2_{\text {eq }}, 2_{\text {ax }}, 4_{\text {ax }}\right), 2.07-1.83$ (m, 2H, cyclobutyl $\mathrm{H}-3_{\text {eq }}, 3_{\text {ax }}$ ); LC-MS, m/z = $293[\mathrm{M}+1]$; Anal. Calcd. for $\mathrm{C}_{13} \mathrm{H}_{16} \mathrm{~N}_{4} \mathrm{O}_{2} \mathrm{~S}: \mathrm{C}, 53.41 ; \mathrm{H}$, 5.52; N, 19.16; S, 10.97; Found: C, 53.47; H, $5.59 ; \mathrm{N}, 19.21 ; \mathrm{S}, 11.03$.

$N$-(2-Isonicotinoylhydrazine-1carbonothioyl)cyclobutanecarboxamide (2.11). Yield: $55 \%$; Mp.: $166-177{ }^{\circ} \mathrm{C} ;{ }^{1} \mathrm{H}$ NMR (400 MHz, DMSO-d ${ }_{6}$ ), $\delta: 12.29$ (s, $1 \mathrm{H},-\mathrm{C}(\mathrm{S}) \mathrm{NH}-$ ), 11.39 (s, 1H, -C(O)NH-), 11.26 (s, 1H, -HN$\mathrm{NH}-\mathrm{C}(\mathrm{O})-), 8.71$ (d, J = 4.9 Hz, 2H, Pyr $\mathrm{H}-3,5), 7.81$ (d, J = 5.0 Hz, 2H, Pyr H-2,6), 3.42 (p, J = 8.6 Hz, $1 \mathrm{H}$, cyclobutyl H-1), 2.39$2.09\left(\mathrm{~m}, 4 \mathrm{H}\right.$, cyclobutyl $\left.\mathrm{H}-4_{\text {eq }}, 2_{\text {eq }}, 2_{\mathrm{ax}}, 4_{\mathrm{ax}}\right)$, 2.07-1.80 (m, 2H, cyclobutyl H-3 $\left.{ }_{\text {eq }}, 3_{\text {ax }}\right)$; LC$\mathrm{MS}, \mathrm{m} / \mathrm{z}=279[\mathrm{M}+1]$; Anal. Calcd. for $\mathrm{C}_{12} \mathrm{H}_{14} \mathrm{~N}_{4} \mathrm{O}_{2} \mathrm{~S}: \mathrm{C}, 51.78 ; \mathrm{H}, 5.07 ; \mathrm{N}, 20.13 ; \mathrm{S}$, 11.52; Found: C, 57.83; H, 5.13; N, 20.19; S, 11.59 .

N-(2-(2-Phenoxyacetyl)hydrazine-1-carbonothioyl)cyclopentanecarboxamide (2.12). Yield: $59 \%$; Mp.: $158-161{ }^{\circ} \mathrm{C}$; ${ }^{1} \mathrm{H}$ NMR (400 MHz, DMSO-d $\mathrm{d}_{6}$ ), $\delta: 12.63$ (s, 1H, -C(S) $\mathrm{NH}-), 11.48$ (s, 1H, -C(O)NH-), 10.81 (br.s, 1H, -HN-NH-C(O)-), 7.31-7.23 (m, 2H, Ph3,5), 7.04-6.92 (m, 3H, Ph-2,4,6), 4.67 (s, 2H, $\left.-\mathrm{CH}_{2} \mathrm{OPh}\right), 3.23-3.11(\mathrm{~m}, 1 \mathrm{H}$, cyclopentyl $\mathrm{H}-1), 1.94-1.51$ (m, 8H, cyclopentyl $\mathrm{H}-5_{\mathrm{eq}}$, $\left.2_{\text {eq }}, 5_{\text {ax }}, 2_{\text {ax }}, 3_{\text {eq }}, 4_{\text {eq }}, 3_{\text {ax }}, 4_{\text {ax }}\right)$; LC-MS, m/z $=322[\mathrm{M}+1]$; Anal. Calcd. for $\mathrm{C}_{15} \mathrm{H}_{19} \mathrm{~N}_{3} \mathrm{O}_{3} \mathrm{~S}$ :
C, 56.06; H, 5.96; N, 13.07; S, 9.98; Found: C, 56.12; H, 6.02; N, 13.13; S, 10.04 .

$\mathrm{N}$-(2-(2-(Phenylthio)acetyl) hydrazine1-carbonothioyl)cyclopentanecarboxamide (2.13). Yield: $63 \%$; Mp.: $148-150{ }^{\circ} \mathrm{C} ;{ }^{1} \mathrm{H}$ NMR (400 MHz, DMSO-d d $_{6}, \delta: 12.52$ (br. s, $1 \mathrm{H},-\mathrm{C}(\mathrm{S}) \mathrm{NH}-), 11.51(\mathrm{~s}, 1 \mathrm{H},-\mathrm{C}(\mathrm{O}) \mathrm{NH}-)$, $10.05(\mathrm{~s}, 1 \mathrm{H},-\mathrm{HNNHC}(\mathrm{O})-), 7.39$ (d, J = $7.7 \mathrm{~Hz}, 2 \mathrm{H}, \mathrm{Ph} \mathrm{H}-2,6), 7.31$ (t, J = 7.7 Hz, 2H, $\mathrm{Ph} \mathrm{H}-3,5), 7.20$ (m, 1H, Ph H-4), 3.84 (s, 2H, $\left.-\mathrm{CH}_{2} \mathrm{SPh}\right), 3.42-3.36(\mathrm{~m}, 1 \mathrm{H}$, cyclopentyl $\mathrm{H}-1), 2.21-2.10\left(\mathrm{~m}, 2 \mathrm{H}\right.$, cyclopentyl $\mathrm{H}-5_{\text {eq }}$, $\left.2_{\mathrm{eq}}\right), 1.86-1.49$ ( $\mathrm{m}, 6 \mathrm{H}$ cyclopentyl H-5 $5_{\mathrm{ax}}, 2_{\mathrm{ax}}$, $\left.3_{\text {eq }}, 4_{\text {eq }}, \mathrm{H}-3_{\text {ax }}, 4_{\text {ax }}\right) ; \mathrm{LC}-M S, \mathrm{~m} / \mathrm{z}=338[\mathrm{M}+1]$; Anal. Calcd. for $\mathrm{C}_{15} \mathrm{H}_{19} \mathrm{~N}_{3} \mathrm{O}_{2} \mathrm{~S}_{2}: \mathrm{C}, 53.39 ; \mathrm{H}$, 5.68; N, 12.45; S, 19.00; Found: C, 53.43; H, $5.73 ; \mathrm{N}, 12.49 ; \mathrm{S}, 19.06$.

$N$-(2-Benzoylhydrazine-1-carbonothioyl) cyclopentanecarboxamide (2.14). Yield: $66 \%$; Mp.: $193-195{ }^{\circ} \mathrm{C}$; ${ }^{1} \mathrm{H}$ NMR $(400 \mathrm{MHz}$, DMSO-d $\left.{ }_{6}\right), \delta: 12.41$ (br.s, $\left.1 \mathrm{H},-\mathrm{C}(\mathrm{S}) \mathrm{NH}-\right)$, 11.46 (s, 1H, - $\mathrm{C}(\mathrm{O}) \mathrm{NH}-), 10.86$ (br.s, $1 \mathrm{H},-\mathrm{HN}-$ $\mathrm{NH}-\mathrm{C}(\mathrm{O})-), 7.91$ (d, J = 7.5 Hz, 2H, Ph-2,6), $7.51(\mathrm{~m}, 3 \mathrm{H}, \mathrm{Ph}-3,4,5), 2.99$ (p, J = 7.9 Hz, $1 \mathrm{H}$, cyclopentyl H-1), 1.95-1.56 (m, 8H, cyclopentyl H-5 eq $, 2_{\text {eq }}, 5_{\mathrm{ax}}, 2_{\mathrm{ax}}, 3_{\mathrm{eq}}, 4_{\mathrm{eq}}, 3_{\mathrm{ax}}, 4_{\mathrm{ax}}$ ); LC-MS, $\mathrm{m} / \mathrm{z}=292[\mathrm{M}+1]$; Anal. Calcd. for $\mathrm{C}_{14} \mathrm{H}_{17} \mathrm{~N}_{3} \mathrm{O}_{2} \mathrm{~S}: \mathrm{C}, 57.71 ; \mathrm{H}, 5.88 ; \mathrm{N}, 14.42 ; \mathrm{S}$, 11.00; Found: C, 57.76; H, 5.93; N, 14.48; S, 11.08 .

$N$-(2-(2-Aminobenzoyl)hydrazine-1-carbonothioyl)cyclopentanecarboxamide (2.15). Yield: $56 \%$; Mp.: $195-197{ }^{\circ} \mathrm{C}$; ${ }^{1} \mathrm{H}$ NMR (400 MHz, DMSO-d $\mathrm{d}_{6}$ ), $\delta: 12.53$ (s, $1 \mathrm{H},-\mathrm{C}(\mathrm{S})$ $\mathrm{NH}-), 11.44$ (s, 1H, -C(O)NH-), 8.01-7.30 (m, $\left.3 \mathrm{H}, \mathrm{Ar} \mathrm{H}-6, \mathrm{NH}_{2}\right), 7.15$ (t, J = 7.5 Hz, $1 \mathrm{H}, \mathrm{Ar}$ $\mathrm{H}-4), 6.72(\mathrm{~d}, \mathrm{~J}=8.3 \mathrm{~Hz}, 1 \mathrm{H}, \mathrm{Ar} \mathrm{H}-3), 6.53$ $(\mathrm{t}, \mathrm{J}=7.5 \mathrm{~Hz}, 1 \mathrm{H}, \mathrm{Ar} \mathrm{H}-5), 3.03-2.93(\mathrm{~m}, 1 \mathrm{H}$, cyclopentyl H-1), 1.96-1.55 (m, 8H, cyclopen- 
tyl H-5 eq $\left., 2_{\text {eq }}, 5_{\mathrm{ax}}, 2_{\mathrm{ax}}, 3_{\mathrm{eq}}, 4_{\mathrm{eq}}, 3_{\mathrm{ax}}, 4_{\mathrm{ax}}\right)$; LC$\mathrm{MS}, \mathrm{m} / \mathrm{z}=307[\mathrm{M}+1]$; Anal. Calcd. for $\mathrm{C}_{14} \mathrm{H}_{18} \mathrm{~N}_{4} \mathrm{O}_{2} \mathrm{~S}: \mathrm{C}, 54.88 ; \mathrm{H}, 5.92 ; \mathrm{N}, 18.29 ; \mathrm{S}$, 10.46; Found: C, 54.93; H, 5.98; N, 18.32; S, 10.51 .

$N$-(2-(Furan-2-carbonyl)hydrazine-1-carbonothioyl)cyclopentanecarboxamide (2.16). Yield: $63 \%$; Mp.: 213-216 ${ }^{\circ} \mathrm{C}$; ${ }^{1} \mathrm{H}$ NMR (400 MHz, DMSO-d $\left.)_{6}\right), \delta: 12.47$ (s, $1 \mathrm{H},-\mathrm{C}(\mathrm{S})$ NH-), 11.39 (s, 1H, -C(O)NH-), 10.55 (s, 1H, -HN-NH-C(O)-), 7.73 (br.s, 1H, furyl H-5), $7.21(\mathrm{~d}, \mathrm{~J}=3.6 \mathrm{~Hz}, 1 \mathrm{H}$, furyl $\mathrm{H}-3), 6.58-6.51$ (m, 1H, furyl $\mathrm{H}-4), 2.98(\mathrm{~m}, 1 \mathrm{H}$, cyclopentyl $\mathrm{H}-1), 1.85-1.60$ (m, 4H, cyclopentyl $\mathrm{H}-5_{\text {eq }}$, $2_{\text {eq }}, \mathrm{H}-5_{\mathrm{ax}}, 2_{\mathrm{ax}}$ ), 1.43-1.14 (m, 4H cyclopentyl $\left.3_{\text {eq }}, 4_{\text {eq }}, \mathrm{H}-3_{\mathrm{ax}}, 4_{\mathrm{ax}}\right)$; Anal. Calcd. for $\mathrm{C}_{12} \mathrm{H}_{15} \mathrm{~N}_{3} \mathrm{O}_{3} \mathrm{~S}$ : C, 51.23; H, 5.37; N, 14.94; $\mathrm{S}$, 11.40; Found: C, 51.28; H, 5.41; N, 15.01; S, 11.48 .

$N$-(2-Thiophene-2-carbonyl)hydrazine1-carbonothioyl)cyclopentanecarboxamide (2.17). Yield: $43 \%$; Mp.: $155-157{ }^{\circ} \mathrm{C} ;{ }^{1} \mathrm{H}$ NMR (400 MHz, DMSO-d $\left.{ }_{6}\right), \delta$ : 12.20 (br.s, $1 \mathrm{H},-\mathrm{C}(\mathrm{S}) \mathrm{NH}-), 11.33$ (s, 1H, - $\mathrm{C}(\mathrm{O}) \mathrm{NH}-)$, 10.83 (br.s, $1 \mathrm{H},-\mathrm{HN}-\mathrm{NH}-\mathrm{C}(\mathrm{O})-), 7.85$ (d, J = $3.8 \mathrm{~Hz}, 1 \mathrm{H}$, thienyl $\mathrm{H}-3), 7.64(\mathrm{~d}, \mathrm{~J}=4.9 \mathrm{~Hz}$, $1 \mathrm{H}$, thienyl H-5), $7.09(\mathrm{t}, \mathrm{J}=4.4 \mathrm{~Hz}, 1 \mathrm{H}$, thienyl H-4), 3.01-2.97 (m, 1H, cyclopentyl H-1), 1.84-1.60 (m, $4 \mathrm{H}$, cyclopentyl $\mathrm{H}-5_{\mathrm{eq}}, 2_{\mathrm{eq}}$, $\left.\mathrm{H}-5_{\mathrm{ax}}, 2_{\mathrm{ax}}\right), 1.42-1.15\left(\mathrm{~m}, 4 \mathrm{H}\right.$, cyclopentyl $3_{\text {eq, }}$, $4_{\text {eq }}, \mathrm{H}-3_{\text {ax }}, 4_{\mathrm{ax}}$ ); Anal. Calcd. for $\mathrm{C}_{12} \mathrm{H}_{15} \mathrm{~N}_{3} \mathrm{O}_{2} \mathrm{~S}_{2}$ : C, 48.47; H, 5.08; N, 14.13; S, 21.56; Found: C, 48.52; H, 5.13; N, 14.18; S, 21.61.

$N$-(2-Isonicotinoylhydrazine - 1 carbonothioyl)cyclopentanecarboxamide (2.18). Yield: 54 \%; Mp.: $185-186{ }^{\circ} \mathrm{C} ;{ }^{1} \mathrm{H}$ NMR (400 MHz, DMSO-d ${ }_{6}$ ), $\delta: 12.34$ (br.s, 1H, -C(S)NH-), 11.47 (s, 1H, -C(O)NH-), 11.21 (br.s, $1 \mathrm{H}$, -HN-NH-C(O)-), 8.69 (d, J = 5.2 Hz,
2H, Pyr H -3,5), 7.79 (t, J = 4.8 Hz, 2H, Pyr H $-2,6), 3.06-2.94(\mathrm{~m}, 1 \mathrm{H}$, cyclopentyl $\mathrm{H}-1)$, $1.95-1.55\left(\mathrm{~m}, 8 \mathrm{H}\right.$, cyclopentyl H-5 $5_{\mathrm{eq}}, 2_{\mathrm{eq}}, 5_{\mathrm{ax}}$, $\left.2_{\mathrm{ax}}, 3_{\mathrm{eq}}, 4_{\mathrm{eq}}, 3_{\mathrm{ax}}, 4_{\mathrm{ax}}\right) ; \mathrm{LC}-\mathrm{MS}, \mathrm{m} / \mathrm{z}=293[\mathrm{M}+1]$; Anal. Calcd. for $\mathrm{C}_{13} \mathrm{H}_{16} \mathrm{~N}_{4} \mathrm{O}_{2} \mathrm{~S}: \mathrm{C}, 53.41 ; \mathrm{H}$, 5.52; N, 19.16; S, 10.97; Found: C, 53.48; H, $5.59 ; \mathrm{N}, 19.21 ; \mathrm{S}, 11.03$.

$N$-(2-(2-Phenoxyacetyl) hydrazine-1-carbonothioyl)cyclohexanecarboxamide (2.19). Yield: $89 \%$; Mp.: $160-162{ }^{\circ} \mathrm{C} ;{ }^{1} \mathrm{H}$ NMR (400 MHz, DMSO-d ${ }_{6}$ ), $\delta: 12.29$ (s, 1H, C(S)NH-), 11.48 (s, 1H, -C(O)NH-), 10.90 (s, 1H, -HNNH-C(O)-), 7.32-7.27 (m, 2H, Ph H-3,5), 6.98 (m, 3H, Ph H-2,4,6), 4.67 (s, 2H, $-\mathrm{CH}_{2} \mathrm{OPh}$ ), 2.55-2.50 (m, 1H, cyclohexyl H-1), 1.79-1.57 $\left(\mathrm{m}, 5 \mathrm{H}\right.$, cyclohexyl $\left.\mathrm{H}-6_{\text {eq }}, 2_{\text {eq }}, 3_{\text {eq }}, 5_{\text {eq }}, 6_{\text {ax }}\right)$, 1.40-1.10 (m, 5H, cyclohexyl $\mathrm{H}-2_{\mathrm{ax}}, 3_{\mathrm{ax}}, 5_{\mathrm{ax}}$, $\left.4_{\text {eq }}, 4_{\mathrm{ax}}\right)$; LC-MS, m/z $=336[\mathrm{M}+1]$; Anal. Calcd. for $\mathrm{C}_{16} \mathrm{H}_{21} \mathrm{~N}_{3} \mathrm{O}_{3} \mathrm{~S}: \mathrm{C}, 57.29 ; \mathrm{H}, 6.31 ; \mathrm{N}$, 12.53; S, 9.56; Found: C, 57.34; H, 6.39; N, $12.59 ; \mathrm{S}, 9.61$.

N-(2-(2-(Phenylthio) acetyl)hydrazine1-carbonothioyl)cyclohexanecarboxamide (2.20). Yield: $61 \%$; Mp.: $136-138{ }^{\circ} \mathrm{C} ;{ }^{1} \mathrm{H}$ NMR (400 MHz, DMSO-d ${ }_{6}$ ), $\delta: 12.50$ (br. s, $1 \mathrm{H},-\mathrm{C}(\mathrm{S}) \mathrm{NH}-), 11.45$ (s, $1 \mathrm{H},-\mathrm{C}(\mathrm{O}) \mathrm{NH}-)$, 11.10 (br.s, 1H, -HNNHC(O)-), 7.32 (d, J = $7.7 \mathrm{~Hz}, 2 \mathrm{H}, \mathrm{Ph} \mathrm{H}-2,6), 7.32$ (t, J = 7.7 Hz, 2H, Ph H-3,5), 7.19 (t, J = 7.4 Hz, 1H, Ph H-4), $3.83\left(\mathrm{~s}, 2 \mathrm{H},-\mathrm{CH}_{2} \mathrm{SPh}\right), 2.55-2.49(\mathrm{~m}, 1 \mathrm{H}$, cyclohexyl H-1), 1.82-1.57 (m, 5H, cyclohexyl H-6 $6_{\text {eq }}, 2_{\text {eq }}, 3_{\text {eq }}, 5_{\text {eq }}, 6_{\text {ax }}$ ), 1.39-1.09 (m, $5 \mathrm{H}$, cyclohexyl H-2 $\left.2_{\mathrm{ax}}, 3_{\mathrm{ax}}, 5_{\mathrm{ax}}, 4_{\mathrm{eq}}, 4_{\mathrm{ax}}\right)$; LC$\mathrm{MS}, \mathrm{m} / \mathrm{z}=352[\mathrm{M}+1]$; Anal. Calcd. for $\mathrm{C}_{16} \mathrm{H}_{21} \mathrm{~N}_{3} \mathrm{O}_{2} \mathrm{~S}_{2}$ : C, 54.68; H, 6.02; N, 11.96; O, 9.10; S, 18.24; Found: C, 54.72; H, 6.07; N, $12.02 ; \mathrm{S}, 18.29$.

$N$-(2-benzoylhydrazine-1-carbonothioyl) cyclohexanecarboxamide (2.21). Yield: $48 \%$; 
Mp.: $183-185{ }^{\circ} \mathrm{C}$; ${ }^{1} \mathrm{H}$ NMR (400 MHz, DMSO-d $\left.{ }_{6}\right), \delta: 12.47$ (s, 1H, -C(S)NH-), 11.37 (s, 1H, -C(O)NH-), $10.80(\mathrm{~s}, 1 \mathrm{H},-\mathrm{HN}-\mathrm{NH}-$ $\mathrm{C}(\mathrm{O})-), 7.90$ (d, J = 7.6 Hz, 2H, Ph-2,6), 7.577.42 (m, 3H, Ph H-3,4,5), 2.55 (p, J = 8.4 Hz, 1H, , cyclohexyl H-1), 1.89-1.64 (m, 5H, cyclohexyl H-6 $\left.6_{\text {eq }}, 2_{\text {eq }}, 3_{\text {eq }}, 5_{\text {eq }}, 6_{\text {ax }}\right), 1.48-1.17$ (m, $5 \mathrm{H}$, cyclohexyl H-2 $\left.2_{\mathrm{ax}}, 3_{\mathrm{ax}}, 5_{\mathrm{ax}}, 4_{\mathrm{eq}}, 4_{\mathrm{ax}}\right)$; LC$\mathrm{MS}, \mathrm{m} / \mathrm{z}=306[\mathrm{M}+1]$; Anal. Calcd. for $\mathrm{C}_{15} \mathrm{H}_{19} \mathrm{~N}_{3} \mathrm{O}_{2} \mathrm{~S}$ : C, 58.99; H, 6.27; N, 13.76; S, 10.50; Found: C, 59.05; H, 6.32; N, 13.81; S, 10.58 .

N-(2-(4-(Tert-butyl)benzoyl)hydrazine1-carbonothioyl)cyclohexanecarboxamide (2.22). Yield: $81 \%$; Mp.: $170-175{ }^{\circ} \mathrm{C} ;{ }^{1} \mathrm{H}$ NMR (400 MHz, DMSO-d $)$ ), $\delta: 12.46$ (s, 1H, $\mathrm{C}(\mathrm{S}) \mathrm{NH}-), 11.33$ (s, 1H, -C(O)NH-), 10.66 (s, 1H, -HN-NH-C(O)-), 7.79 (d, J = 8.1 Hz, 2H, Ph-2,6), 7.42 (d, J = 8.1 Hz, 2H, Ph-3,5), 2.562.47 (m, 1H, cyclohexyl H-1), 1.84-1.60 (m, $5 \mathrm{H}$, cyclohexyl H-6 $\left.6_{\text {eq }}, 2_{\text {eq }}, 3_{\text {eq }}, 5_{\text {eq }}, 6_{\text {ax }}\right), 1.46-$ $1.10\left(\mathrm{~m}, 14 \mathrm{H}\right.$, cyclohexyl $\mathrm{H}-2_{\mathrm{ax}}, 3_{\mathrm{ax}},-\mathrm{C}\left(\mathrm{CH}_{3}\right)_{3}$, $\left.5_{\mathrm{ax}}, 4_{\mathrm{eq}}, 4_{\mathrm{ax}}\right)$; LC-MS, m/z = 362 [M+2]; Anal. Calcd. for $\mathrm{C}_{19} \mathrm{H}_{27} \mathrm{~N}_{3} \mathrm{O}_{2} \mathrm{~S}: \mathrm{C}, 63.13 ; \mathrm{H}, 7.53 ; \mathrm{N}$, 11.62, S, 8.87; Found: C, 63.21; H, 7.60; N, 11.69; S, 8.93.

N-(2-(2-Aminobenzoyl)hydrazine-1-carbonothioyl)cyclohexanecarboxamide (2.23). Yield: $49 \%$; Mp.: 213-216 ${ }^{\circ} \mathrm{C} ;{ }^{1} \mathrm{H}$ NMR (400 MHz, DMSO-d 6 ), $\delta: 12.62(\mathrm{~s}, 1 \mathrm{H},-\mathrm{C}(\mathrm{S})$ NH-), 11.34 (s, 1H, -C(O)NH-), 8.18-7.30 (m, $\left.3 \mathrm{H}, \mathrm{Ar} \mathrm{H}-6, \mathrm{NH}_{2}\right), 7.15$ (t, J = 7.7 Hz, 1H, Ar H-4), 6.72 (d, J = 8.3 Hz, 1H, Ar H-3), 6.53 (t, J = 7.5 Hz, 1H, Ar H-5), 5.30 (d, J = 4.5 Hz, $\left.2 \mathrm{H},-\mathrm{NH}_{2}\right), 2.60-2.46(\mathrm{~m}, 1 \mathrm{H}$, cyclohexyl $\mathrm{H}-1), 1.92-1.60$ (m, 5H, cyclohexyl H-6 ${ }_{\text {eq }}, 2_{\text {eq }}$, $\left.3_{\text {eq }}, 5_{\text {eq }}, 6_{\text {ax }}\right), 1.51-1.14(\mathrm{~m}, 5 \mathrm{H}$, cyclohexyl $\left.\mathrm{H}-2_{\mathrm{ax}}, 3_{\mathrm{ax}}, 5_{\mathrm{ax}}, 4_{\text {eq }}, 4_{\mathrm{ax}}\right)$; LC-MS, m/z $=321$ $[\mathrm{M}+1]$; Anal. Calcd. for $\mathrm{C}_{15} \mathrm{H}_{20} \mathrm{~N}_{4} \mathrm{O}_{2} \mathrm{~S}$ : C,
56.23; H, 6.29; N, 17.49; S, 10.01; Found: C, 56.20; H, 6.31; N, 17.42; S, 9.97.

N-(2-(5-Methylfuran-2-carbonyl)hydrazine1-carbonothioyl)cyclohexanecarboxamide (2.24). Yield: $84 \%$; Mp.: $184-186{ }^{\circ} \mathrm{C} ;{ }^{1} \mathrm{H}$ NMR (400 MHz, DMSO-d 6 ), $\delta: 12.33$ (br.s, 1H, -C(S)NH-), 11.29 (s, 1H, -C(O)NH-), 10.32 (br.s, 1H, -HN-NH-C(O)-), 7.32 (d, J = $2.1 \mathrm{~Hz}, 1 \mathrm{H}$, furyl $\mathrm{H}-3), 6.83(\mathrm{~d}, \mathrm{~J}=2.1 \mathrm{~Hz}$, $1 \mathrm{H}$, furyl $\mathrm{H}-4), 2.53\left(\mathrm{~s}, 3 \mathrm{H},-\mathrm{CH}_{3}\right), 2.53-2.43$ (m, 1H, cyclohexyl H-1), 1.86-1.57 (m, 5H, cyclohexyl H-6 $\left.6_{\text {eq }}, 2_{\text {eq }}, 3_{\text {eq }}, 5_{\text {eq }}, 6_{\text {ax }}\right), 1.42-1.14$ (m, 5H, cyclohexyl H-2 $\left.2_{\mathrm{ax}}, 3_{\mathrm{ax}}, 5_{\mathrm{ax}}, 4_{\mathrm{eq}}, 4_{\mathrm{ax}}\right)$; LC-MS, m/z = 310 [M+1]; Anal. Calcd. for $\mathrm{C}_{14} \mathrm{H}_{19} \mathrm{~N}_{3} \mathrm{O}_{3} \mathrm{~S}: \mathrm{C}, 54.35 ; \mathrm{H}, 6.19 ; \mathrm{N}, 13.58 ; \mathrm{S}$, 10.36; Found: C, 54.39; H, 6.23; N, 13.63; S, 10.41 .

$N-(2-(5-B r o m o f u r a n-2-c a r b o n y l) h y d r a z i n e-$ 1-carbonothioyl)cyclohexanecarboxamide (2.25). Yield: $55 \%$; Mp.: $133-135{ }^{\circ} \mathrm{C} ;{ }^{1} \mathrm{H}$ NMR (400 MHz, DMSO-d 6 ), $\delta: 12.25$ (s, 1H, $\mathrm{C}(\mathrm{S}) \mathrm{NH}-), 11.37$ (s, 1H, -C(O)NH-), 10.76 (s, 1H, -HN-NH-C(O)-), 7.24 (d, J = 3.6 Hz, 1H, furyl $\mathrm{H}-3), 6.58$ (d, J = 3.6 Hz, $1 \mathrm{H}$, furyl $\mathrm{H}-4)$, 2.55-2.43 (m, 1H, cyclohexyl H-1), 1.85-1.56 $\left(\mathrm{m}, 5 \mathrm{H}\right.$, cyclohexyl H-6 $6_{\text {eq }}, 2_{\text {eq }}, 3_{\text {eq }}, 5_{\text {eq }}, 6_{\text {ax }}$ ), 1.43-1.10 (m, 5H, cyclohexyl H-2 $, 3_{\mathrm{ax}}, 5_{\mathrm{ax}}$, $\left.4_{\text {eq }}, 4_{\text {ax }}\right)$; LC-MS, m/z = 376 [M+2]; Anal. Calcd. for $\mathrm{C}_{13} \mathrm{H}_{16} \mathrm{BrN}_{3} \mathrm{O}_{3} \mathrm{~S}$ : C, 41.72; $\mathrm{H}, 4.31$; N, 11.23 S, 8.57; Found: C, 41.79; H, 4.39; N, $11.31 ; \mathrm{S}, 8.62$.

N-(2-Benzoylhydrazine-1-carbonothioyl) adamantane-1-carboxamide (2.26). Yield: $43 \%$; Mp.: $158-160{ }^{\circ} \mathrm{C}$; ${ }^{1} \mathrm{H}$ NMR (400 MHz, DMSO-d $\left.{ }_{6}\right), \delta: 12.64$ (br. s, $\left.1 \mathrm{H},-\mathrm{C}(\mathrm{S}) \mathrm{NH}-\right)$, 11.91 (br. s, 1H, -C(O)NH-), 10.38 (br.s, 1H,HN-NH-C(O)-), 7.96 (d, J = 7.6 Hz, 2H, Ph2,6), 7.53-7.34 (m, 3H, Ph H-3,4,5), 2.17-2.05 (m, $15 \mathrm{H}$, Ad H-3, 5, 7, $2_{\text {eq }}, 8_{\text {eq }}, 9_{\text {eq }}, 2_{\text {ax }}, 8_{\text {ax }}$, 
$\left.9_{\text {ax }}\right), 1.75-1.62\left(\mathrm{~m}, 6 \mathrm{H}\right.$, Ad $4_{\text {eq }}, 6_{\text {eq }}, 10_{\text {eq }}, 4_{\text {ax }}$, $6_{\mathrm{ax}}, 10_{\mathrm{ax}}$ ); Anal. Calcd. for $\mathrm{C}_{19} \mathrm{H}_{23} \mathrm{~N}_{3} \mathrm{O}_{2} \mathrm{~S}: \mathrm{C}$, 63.84; H, 6.49; N, 11.76; S, 8.97; Found: C, 63.91; H, 6.54; N, 11.81; S, 9.03.

N-(2-Isonicotinoylhydrazine-1carbonothioyl)adamantane-1-carboxamide (2.27). Yield: $39 \%$; Mp.: $152-155{ }^{\circ} \mathrm{C} ;{ }^{1} \mathrm{H}$ NMR (400 MHz, DMSO-d $\left.{ }_{6}\right), \delta: 12.44(\mathrm{~s}, 1 \mathrm{H}$, -C(O)NH-), 11.25 (s, 1H, -C(S)NH-), 10.63 (s, 1H, -HN-NH-C(O)-), 8.71 (d, J = 4.9 Hz, 2H, Pyr H-3,5), 7.80 (d, J = 4.9 Hz, 2H, Pyr H-2,6), 2.09-1.61 (m, 15H, Ad); LC-MS, $\mathrm{m} / \mathrm{z}=359$ [M+1]; Anal. Calcd. for $\mathrm{C}_{18} \mathrm{H}_{22} \mathrm{~N}_{4} \mathrm{O}_{2} \mathrm{~S}$ : C, 60.31; H, 6.19; N, 15.63; S, 8.94; Found: C, $60.38 ; \mathrm{H}, 6.23 ; \mathrm{N}, 15.68 ; \mathrm{S}, 9.03$.

Molecular docking. Research was conducted by flexible molecular docking, as an approach of finding the molecules with affinity to a specific biological target. Macromolecules from Protein Data Bank (PDB) were used as biological targets, namely human $\mathrm{GABA}_{\mathrm{A}}$ receptor alpha 1 -beta - -gamma $_{2}$ subtype (PDB ID - 6X3W), 4-aminobutyrate-aminotransferase inactivated by gamma-vinyl $\mathrm{GABA}_{\mathrm{T}}$ (PDB ID - 1OHW) and full length Wild-Type Open-form Sodium Channel NavMs (NavMs, PDB ID - 5HVX) [Protein Data Bank. http://www.rcsb.org/pdb/home/ home.-do. Accessed September 6, 2020]. The choice of biological targets was due to the literature on the mechanism of antiepileptic drugs activity $[1,5,21,22]$.

Ligand preparation. The substances were drawn using MarvinSketch 20.20.0 and saved in mol format [MarvinSketch version 20.20.0, ChemAxon http://www.chemaxon. com]. After that they were optimized by program Chem $3 \mathrm{D}$, using molecular mechanical MM2 algorithm, and saved as pdb-files.
Molecular mechanics was used to produce more realistic geometry values for most organic molecules, owing to the fact of being highly parameterized. Using AutoDockTools-1.5.6, the pdb-files were converted into PDBQT, number of active torsions was set as default [23].

Protein preparation. PDB files were downloaded from the protein data bank. Discovery Studio v 19.1.0.18287 was used to delete water molecules and ligands. Structures of proteins were saved as pdb-files [Discovery Studio Visualizer v19.1.018287. Accelrys Software Inc., https://www.3dsbiovia.com]. In AutoDockTools-1.5.6 polar hydrogens were added and saved as PDBQT. Grid box was set as following: center_x $=95.250$, center_y $=$ $=-125.333$, center_ $z=107.722$, size $\mathrm{x}=20$, size $y=20$, size_ $z=20$ for GABAA receptor $(6 \mathrm{X} 3 \mathrm{~W})$; center_x $=8.333$, center_y $=0.222$, center $z=19.806$, size $\mathrm{x}=20$, size $\mathrm{y}=20$, size $z=20$ for GABAT receptor $(1 \mathrm{OHW})$; center_x $=73.556$, center_y $=52.333$, center_z $=25.806$, size $\mathrm{x}=20$, size_ $\mathrm{y}=20$, size $z=20$ for NavMs (5HVX). Vina was used to carry docking [23]. For visualization Discovery Studio v 19.1.0.18287 was used.

Anticonvulsant activity. Estimation of anticonvulsant activity of the synthesized substances was carried out on 114 white rats, the weigh $120-150 \mathrm{~g}$, obtained from the nursery of the Institute of Pharmacology and Toxicology of the Academy of Medical Sciences of Ukraine (Kyiv). The study was conducted under the "Guidelines for the care and use of laboratory animals», published in the United States (by the National Institute of Health [24].

Convulsions were modeled by a single subcutaneous administration of pentylenetetrazole 
(«Nizhpharm», Russian Federation) at a dose of $80 \mathrm{mg} / \mathrm{kg}$ [25]. One hour prior to the administration of the convulsant, the test compounds were administered intragastrically at a dose of $10 \mathrm{mg} / \mathrm{kg}$ as an aqueous suspension stabilized with Tween-80. «Depakine» («Sanofi
Winthrop Industria», France) was used as a reference drug, administered similarly at a dose of $150 \mathrm{mg} / \mathrm{kg}$. The control group of animals intragastrically received a similar volume of water with Tween-80. The determination of the testing time was based on the data on the

Table 1. The results of molecular docking of studied compounds

\begin{tabular}{|c|c|c|c|}
\hline Compd. & $\begin{array}{l}\text { Affinity (kcal/mol) to human } \\
\text { GABA }_{\mathrm{A}} \text { receptor }(6 \mathrm{X} 3 \mathrm{~W})\end{array}$ & $\begin{array}{l}\text { Affinity (kcal/mol) to human } \\
\text { GABA }_{\mathrm{T}} \text { receptor (1OHW) }\end{array}$ & $\begin{array}{l}\text { Affinity (kcal/mol) to } \\
\text { NavMs (5HVX) }\end{array}$ \\
\hline Phenobarbital & -7.4 & & - \\
\hline Valproic acid & - & -5.2 & -4.9 \\
\hline Topiramate & -5.1 & - & -4.7 \\
\hline Gabapentine & -6.1 & - & -4.2 \\
\hline Lamotrigine & - & - & -5.7 \\
\hline Carbamazepine & - & - & -6.5 \\
\hline 2.1 & -6.0 & -6.9 & -4.6 \\
\hline 2.2 & -6.3 & -7.8 & -5.4 \\
\hline 2.3 & -5.8 & -7.8 & -5.2 \\
\hline 2.4 & -5.7 & -7.9 & -4.9 \\
\hline 2.5 & -6.7 & -7.8 & -6.8 \\
\hline 2.6 & -7.0 & -7.6 & -5.0 \\
\hline 2.7 & -6.3 & -8.3 & -5.9 \\
\hline 2.8 & -6.1 & -8.0 & -5.8 \\
\hline 2.9 & -6.5 & -7.6 & -6.3 \\
\hline 2.10 & -6.9 & -8.5 & -5.1 \\
\hline 2.11 & -6.3 & -8.0 & -5.8 \\
\hline 2.12 & -7.2 & -6.8 & -5.9 \\
\hline 2.13 & -6.5 & -6.7 & -5.5 \\
\hline 2.14 & -6.8 & -6.6 & -6.2 \\
\hline 2.15 & -6.4 & -7.8 & -6.4 \\
\hline 2.16 & -6.6 & $\begin{array}{l}-8.3 \\
\end{array}$ & -5.0 \\
\hline 2.17 & -6.2 & $\begin{array}{l}-7.8 \\
\end{array}$ & -5.3 \\
\hline 2.18 & -6.5 & -8.5 & -5.7 \\
\hline 2.19 & -6.6 & -8.4 & -5.9 \\
\hline 2.20 & -6.5 & -8.6 & -6.0 \\
\hline 2.21 & $\begin{array}{l}-7.0 \\
\end{array}$ & $\begin{array}{l}-8.7 \\
\end{array}$ & -5.6 \\
\hline 2.22 & $\begin{array}{l}-6.8 \\
\end{array}$ & $\begin{array}{l}-8.7 \\
\end{array}$ & -6.6 \\
\hline 2.23 & -7.2 & -8.8 & -6.4 \\
\hline 2.24 & -6.3 & -8.5 & -5.8 \\
\hline 2.25 & -6.8 & -8.2 & -4.6 \\
\hline 2.26 & $\begin{array}{l}-7,5 \\
\end{array}$ & $-8,4$ & $-6,3$ \\
\hline 2.27 & -7.1 & -8.5 & -6.3 \\
\hline
\end{tabular}


peak of anticonvulsant activity of the test compounds. The severity of the anticonvulsant effect was evaluated by the duration of the latent period of convulsion, the type and duration of convulsions in minutes and the mortality index. The intensity of the convulsion was assessed using a five-point scale: 0 - no convulsive activity; 1 - hyperkinesia; 2 - trembling, twitching; 3 - clonic spasms of the forepaws with lifting on the hind legs; 4 pronounced tonic-clonic convulsions, falling of the animal on its side, the presence of a phase of tonic extension; 5 - repeated clonictonic convulsions, loss of posture, death.

Statistical data processing was performed using a license program «STATISTICA $®$ for Windows 6.0» (StatSoftInc., № AXXR712D833214FAN5) and «SPSS 16.0», «Microsoft Office Excel 2003». The results are presented as mean \pm standard error of the mean. Arithmetic mean and standard error of the mean were calculated for each of the studied parameters. During verification of statistical hypothesis, null hypothesis was declined if statistical criterion was $\mathrm{p}<0.05$ [26].

\section{Results and Discussion}

\section{Molecular docking}

Molecular docking was used in the first step of our study, as a tool for predicting the affinity of antiepileptic drugs (Phenobarbital, Valproic acid, Lamotrigine, Topiramate, Gabapentin and Carbamazepine) and diacylthiosemicarbazides (2) to active centers of $\mathrm{GABA}_{\mathrm{A}^{-}}, \mathrm{GABA}_{\mathrm{T}}$-receptors and NavMs (Table 2). The results of molecular docking showed, that the planned structural modification of diacylthiosemicarbazides (2) can be justified. Thus, the affinity of compounds 2 was significantly higher for $\mathrm{GABA}_{\mathrm{T}}$ receptor inhibitors and in most cases was higher or comparable to NavMs, than the reference compounds (valproic acid, lamotrigine and carbamazepine).

This was also confirmed by the visualization of the results of molecular docking for drugs and promising compounds. Compounds 2.5 as well as the phenobarbital inhibitor, have the highest binding affinity to the $\mathrm{GABA}_{\mathrm{A}}$ receptor. Thus, compound $\mathbf{2 . 5}$ in the active center of the receptor has two strong hydrogen bonds of Nitrogen atoms of the 2-aminobenzoylhydrazide group with SER E: 301 (2.21 $\AA$ ) and LEU A: $223(2.75 \AA)$, as well as p-donor hydrogen bond of the 2-aminobenzene moiety with SER E: 301 (3.37 Å). (Fig. 2). Moreover, compound 2.5 is characterized by additional hydrophobic alkyl and $\mathrm{N}$-alkyl interactions of cyclopropane and benzene moieties with PHE E: 304 (4.44 $\AA)$, PRO A: 228 (4.97 $\AA)$ and MET A: 227 (5.36 $\AA$ ), respectively.

The main array of compounds (2.2-2.11, 2.15-2.27) has a greater affinity for the $G_{A B A}$ receptor. For example, compounds $\mathbf{2 . 1 5}$ with an active receptor center are predicted to form twice as many hydrogen bonds and other types of interactions (hydrophobic, $\mathrm{N}$-alkyl, attractive) as valproic acid (Fig. 3). Thus, compound 2.15 forms strong hydrogen bonds between the Nitrogen and Oxygen atoms of the 2-aminobenzoylhydrazide group with THR B: 353 $(2.74 \AA)$ and ASN B: 352 (3.27 $\AA$ ), the hydrogen atom of the amino group in the 2-aminobenzyl moiety with THR B: 353 (2.25 $\AA$ ). In addition to these interactions, compound $\mathbf{2 . 1 5}$ has additional weak hydrogen bonds of the sulfur atom of the thioamide group with SER A: 137 (4.26 ^), hydrophobic p-alkyl 
$A$

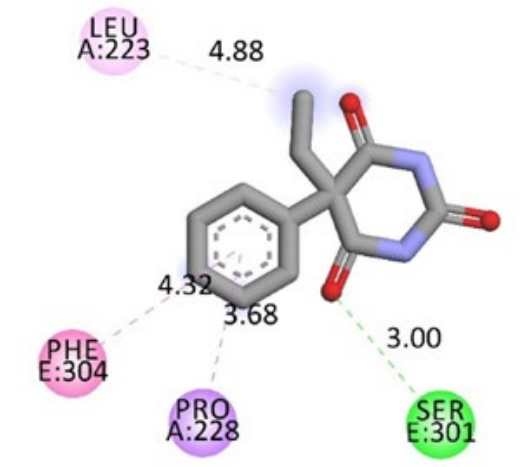

Interactions

Conventional Hydrogen Bond

Pi-Sigma
Pi-Pi Stacked Alkyl

$\boldsymbol{B}$

Phenobarbital

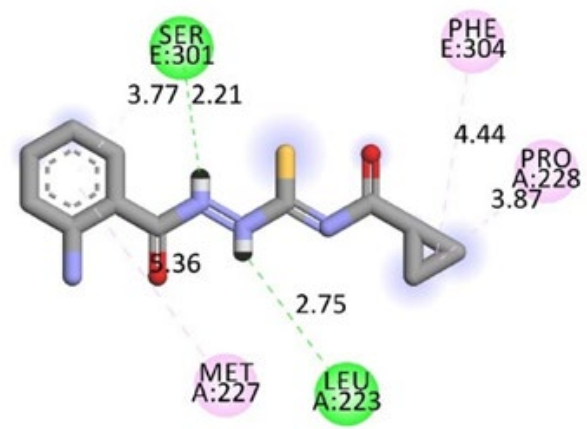

Interactions

Conventional Hydrogen Bond

Pi-Donor Hydrogen Bond

Alkyl

Pi-Alkyl

2.5

Fig. 2. Visualization of affinity according to the docking. $A-$ Phenobarbital with $\mathrm{GABA}_{\mathrm{A}}, B-$ compound $\mathbf{2 . 5}$ with $\mathrm{GABA}_{\mathrm{A}}$.

$\boldsymbol{A}$
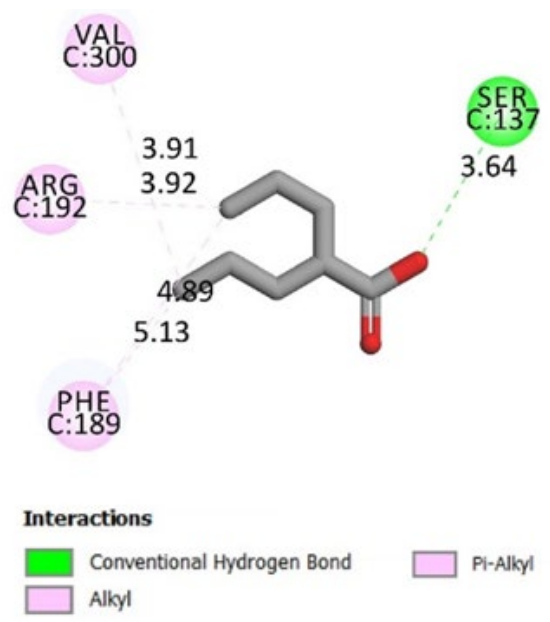

Valproic acid
$\boldsymbol{B}$

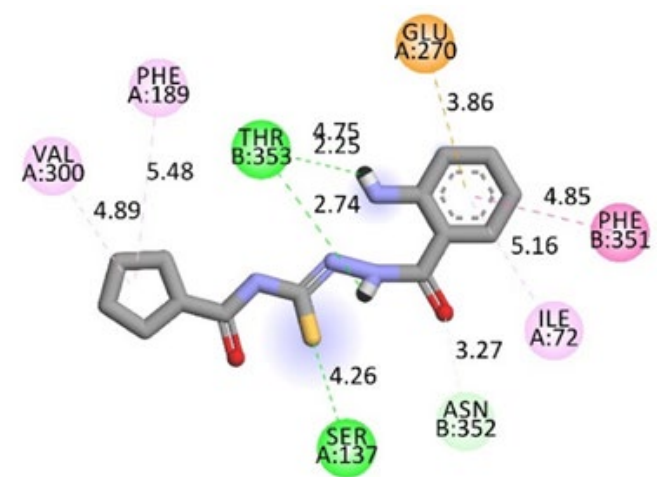

Interactions

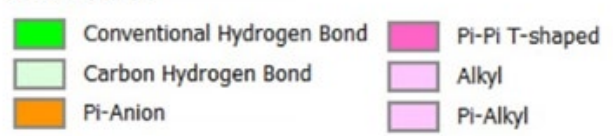

2.15

Fig. 3. Visualization of affinity according to the docking. $A-$ Valproic acid with $\mathrm{GABA}_{\mathrm{T}}, B-$ compound 2.15 with $\mathrm{GABA}_{\mathrm{T}}$.

interactions of the cyclopropane moiety with shaped interactions of the phenyl ring with VAL A: 300 (4.89 $\AA)$ and PHE A: $189(5.48 \AA) \quad$ GLU A: 121 (3.86 $\AA)$, with ILE A: $72(5.16 \AA)$ and p-anionic, hydrophobic alkyl and p-p T and PHE B: 351 (4.85) $\AA$ ). It is important that, 
compounds 2.10, 2.18 and 2.23 also interact with similar amino acid residues but have other types of interactions.

Visualization of the structure $\mathbf{2 . 1 8}$ with the active site of NavMs (Fig. 4) allowed us to establish that the structure has the interactions similar to those existing between Carbamazepine and the active site. Visualization was characterized by a strong hydrogen bond with VAL A: 197 (2.96 A), hydrophobic interactions (p-alkyl) of the cyclopropane moiety with LEU A: $152(5.08 \AA)$ and VAL A: 197 (5.12 $\AA$ ), hydrophobic alkyl and $p-p$ T shaped interactions of the phenyl ring with ALA A: 145 (4.17 $\AA$ ), VAL A: $141(5.43 \AA)$ and PHE A: $198(5.53 \AA)$. Similar types of interactions are also characteristic for valproic acid and compounds $\mathbf{2 . 1 0}$, 2.15, 2.22 and 2.23 with the active site of NavMs.

Thus, the conducted molecular docking and visualization of its results showed the prospects of synthesis and structural modification of diacylthiosemicarbazides (2) for the tar- geted search for anticonvulsants in this class of compounds. It is important that, the anticonvulsant mechanism for these derivatives is predicted to be similar to the mechanism of valproic acid $\left(\mathrm{GABA}_{\mathrm{T}}\right.$ receptor inhibitor and NavMs blocker) [21, 22].

\section{Chemistry}

For further implementation of the research design, we have used cycloalkanecarbonyl isothiocyanates, heterocumulenes with an electrophilic center on the carbon atom, which are characterized by the nucleophile addition reactions. The starting cycloalkanecarbonyl isocyanates were obtained by a known synthetic approach. It included the synthesis of cycloalkanecarbonyl chlorides (1.1-1.5) and their subsequent interaction with ammonium isothiocyanate (acetonitrile medium) [19, 20]. The latter, without isolation from the reaction medium (in situ method), reacted regioselectively and quite easily with hydrazides of cycloalkyl- (aralkyl-, aryl-, hetaryl-) carboxylic

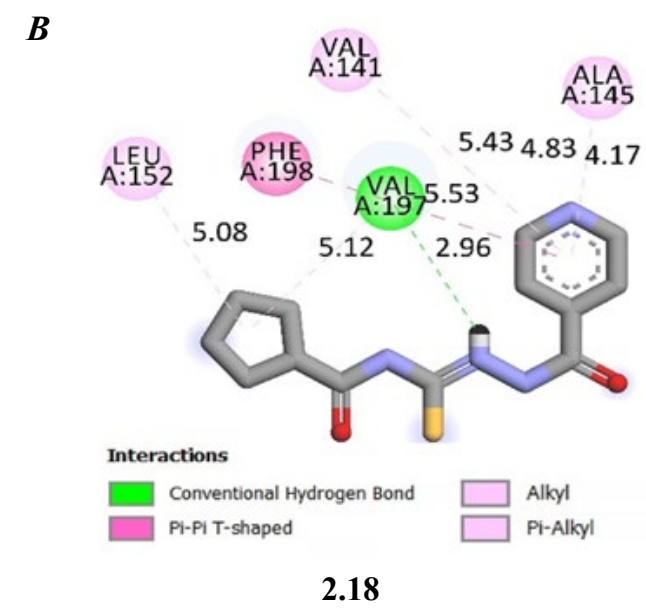

Fig. 4. Visualization of affinity according to the docking. $A$ - Carbamazepine with NavMs, $B-$ compound 2.18 with NavMs. 


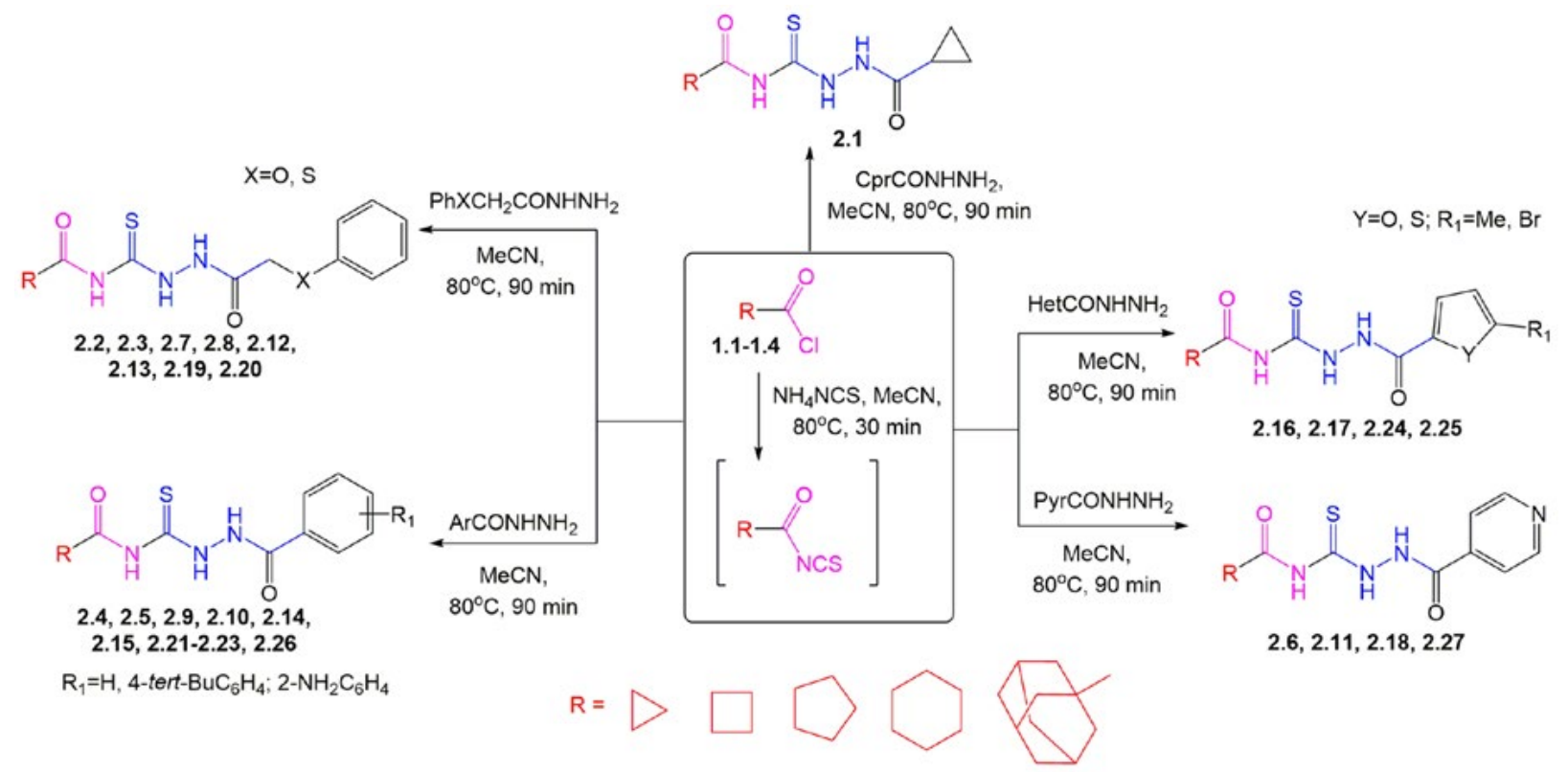

Scheme 1. Synthesis of new diacylthiosemicarbazides.

acids (Scheme 1). Thus, individual diacylthiosemicarbazides (2.1-2.27) are produced with satisfactory yields (39-89\%).

The data of ${ }^{1} \mathrm{H}$ NMR spectra indicated the formation of compounds 2 . Thus, singlet or broader singlet signals of protons of three amide groups: thioamide $(-\mathrm{C}(\mathrm{S}) \mathrm{N} H)$ at the $13.78-$ $12.20 \mathrm{ppm}$, amide $(-\mathrm{C}(\mathrm{O}) \mathrm{N} H-)$ at the $12.58-$ $11.21 \mathrm{ppm}$ and hydrazide $(-\mathrm{HN}-\mathrm{N} H-\mathrm{C}(\mathrm{O})-)$ at the 11.20-10.05 ppm were recorded. An interesting aspect of the ${ }^{1} \mathrm{H}$ NMR spectra of compounds 2.5, 2.10, 2.15 and 2.23 is the absence of a proton signal of the hydrazide group and the presence of a broad $\mathrm{NH}_{2}$ group multiple in the o-position of the phenyl substituent. That is probably due to the hydrogen bonding between these fragments. Additionally, in the ${ }^{1} \mathrm{H}$ NMR spectra of compounds 2 there are proton signals of cycloalkane fragments. They appear in a strong field as wide multiplets of sequen- tially arranged signals of axial and equatorial protons [20]. For compounds 2.1-2.25, the methylene proton of the cycloalkane fragment is registered as a pentet or an expanded multiplet in the range of 3.42-2.03 ppm. Its different chemical shift can be explained by the conformational features of the cycle [20]. Other protons of the acylhydrazide residue of compounds 2 in the ${ }^{1} \mathrm{H}$ NMR spectra have «classical» multiplicity and chemical shifts, which are in accordance with the proposed structures [27]. Additionally, the structure and individuality of compounds 2 were also confirmed by the chromato-mass spectra, in which the mass of quasimolecular ion $[\mathrm{M}+1]$ corresponded to the calculated mass.

\section{Biological assay}

According to the results of molecular docking, diacylthiosemicarbazides, which contain cy- 
clopropane (2.4-2.6), cyclobutane (2.8-2.10), cyclopentane $(\mathbf{2 . 1 3}-\mathbf{2 . 1 5}, \mathbf{2 . 1 8})$, cyclohexane (2.22) and adamantane (2.26) carboxamide groups with similar fragments $\left(\mathrm{PhO}(\mathrm{S}) \mathrm{CH}_{2}\right.$, $\mathrm{Ph}, o-\mathrm{NH}_{2} \mathrm{C}_{6} \mathrm{H}_{4}$, Pyr, fyrul) on the hydrazide residue (Fig. 1, Scheme 1), were selected by a randomized method for the study on the model of pentylenetetrazole seizures.

The results of the studies showed (Table 2) that the administration of pentylenetetrazole to experimental animals led to the development of epileptic seizures with the expressed tonicclonic phase and subsequent $100 \%$ mortality. Thus, in the control group, the latency period averaged 6.11 minutes, and the duration of tonic-clonic seizures was 9.11 minutes. Convulsions observed in this group of animals had an expressed tonic-clonic character and recurred periodically. The introduction of dia- cylthiosemicarbazides (2) to the animals in experimental group led to an increase in the latency period of seizures by $2.77-7.82$ times. It is important that compounds 2.6, 2.15, 2.22 were close and compound $\mathbf{2 . 1 8}$ was higher in potency than the reference drugs Depakine. The test compounds reduced the duration of tonic-clonic seizures by $1.23-5.59$ minutes and prevented animal mortality by $30-60 \%$ relative to the control group. Compound $\mathbf{2 . 1 8}$ exceeded Depakine.

SAR-analysis showed, that the greatest anticonvulsant activity is characteristic of diacylsemicarbazides, which contain in their structure cyclopropane- $(2.5,2.6)$ and cyclopentane- (2.13-2.15, 2.18) carboxamide fragment. Additionally, a significant effect on the activity is characteristic for the hydrazide fragment in the molecule. Thus, the compounds with

Table 2. Anticonvulsant activity of the synthesized compounds

\begin{tabular}{l|l|c|c|c|c}
\hline № & \multicolumn{1}{|c|}{ Compd. } & $\begin{array}{c}\text { Latent convulsion } \\
\text { period, min }\end{array}$ & $\begin{array}{c}\text { Duration of tonic-clonic } \\
\text { convulsion, min }\end{array}$ & Mortality, $\%$ & $\begin{array}{c}\text { Severity of convulsion in } \\
\text { points }\end{array}$ \\
\hline 1 & Model control & $6.11 \pm 0.32$ & $9.11 \pm 0.52$ & 100 & $8.20 \pm 0.53$ \\
\hline 2 & $\mathbf{2 . 4}$ & $18.40 \pm 1.90^{*}$ & $7.33 \pm 1.50$ & $70^{*}$ & $7.55 \pm 0.65$ \\
\hline 3 & $\mathbf{2 . 5}$ & $28.80 \pm 1.70^{*}$ & $5.77 \pm 1.80$ & $70^{*}$ & $4.87 \pm 0.21^{*}$ \\
\hline 4 & $\mathbf{2 . 6}$ & $32.20 \pm 1.70^{*}$ & $4.11 \pm 1.20$ & $50^{*}$ & $5.55 \pm 0.27$ \\
\hline 5 & $\mathbf{2 . 8}$ & $26.10 \pm 1.10^{*}$ & $5.42 \pm 1.20$ & $60^{*}$ & $5.71 \pm 0.28$ \\
\hline 6 & $\mathbf{2 . 9}$ & $17.20 \pm 1.20^{*}$ & $7.11 \pm 1.20$ & $70^{*}$ & $6.11 \pm 0.54$ \\
\hline 7 & $\mathbf{2 . 1 0}$ & $29.20 \pm 1.80^{*}$ & $7.11 \pm 1.00$ & $60^{*}$ & $6.33 \pm 0.42$ \\
\hline 8 & $\mathbf{2 . 1 3}$ & $27.20 \pm 1.30^{*}$ & $5.55 \pm 1.80$ & $50^{*}$ & $5.11 \pm 0.34$ \\
\hline 9 & $\mathbf{2 . 1 4}$ & $23.40 \pm 1.20^{*}$ & $5.23 \pm 1.20$ & $60^{*}$ & $5.55 \pm 0.27$ \\
\hline 10 & $\mathbf{2 . 1 5}$ & $35.20 \pm 3.00^{*}$ & $5.51 \pm 0.25^{*}$ & $60^{*}$ & $5.40 \pm 0.37^{*}$ \\
\hline 11 & $\mathbf{2 . 1 8}$ & $47.80 \pm 4.00^{*}$ & $3.52 \pm 0.33^{*}$ & $40^{*}$ & $4.30 \pm 0.32^{*}$ \\
\hline 12 & $\mathbf{2 . 2 2}$ & $34.20 \pm 2.10^{*}$ & $4.75 \pm 0.33^{*}$ & $60^{*}$ & $4.77 \pm 0.23^{*}$ \\
\hline 13 & $\mathbf{2 . 2 3}$ & $17.40 \pm 1.00^{*}$ & $7.66 \pm 1.90$ & $70^{*}$ & $6.25 \pm 0.55$ \\
\hline 14 & $\mathbf{2 . 2 4}$ & $19.20 \pm 1.70^{*}$ & $7.72 \pm 0.82$ & $70^{*}$ & $6.20 \pm 0.33^{*}$ \\
\hline 15 & $\mathbf{2 . 2 5}$ & $16.90 \pm 1.00^{*}$ & $7.46 \pm 1.60$ & $70^{*}$ & $6.12 \pm 0.55$ \\
\hline 16 & $\mathbf{2 . 2 6}$ & $17.70 \pm 1.40^{*}$ & $7.00 \pm 1.50$ & $70^{*}$ & $6.22 \pm 0.55$ \\
\hline 17 & Model control & $6.22 \pm 0.62$ & $7.88 \pm 0.77$ & 100 & $7.30 \pm 0.55$ \\
\hline 18 & Depakine & $41.10 \pm 0.80^{*}$ & $4.71 \pm 0.42^{*}$ & $40^{*}$ & $3.50 \pm 0.75^{*}$ \\
\hline
\end{tabular}

Note. * - significantly $(\mathrm{p} \leq 0.05)$ relative to the control group of rats 
phenylthioacetyl (2.13), benzoyl (2.5, 2.14, 2.15) and isonicotinic acid $(\mathbf{2} .6,2.18)$ moieties in the thiosemicarbazide residue are more active. These functional groups can be considered «key» pharmacophores for revealing the anticonvulsant activity.

\section{Conclusion}

A virtual target-oriented screening, synthesis, and study of diacylthiosemicarbazides for their anticonvulsant activity were performed on the models of pentylenetetrazole seizures in rats. The structure-activity relationship was discussed for further targeted search for effective drugs. New diacylthiosemicarbazides were synthesized by the in situ method. Biological screening showed that diacylthiosemicarbazides with cyclopropane and cyclopentanecarbamide groups demonstrate the anticonvulsant activity that exceeds or competes with the reference drug «Depakine». The most active compound $\mathbf{2 . 1 8}$ was identified for further study of anticonvulsant activity on other models.

\section{Acknowledgements}

The work was carried out on the budgetary theme of the Ministry of Health of Ukraine «Cycloalkylcarbonylisothiocyanates are effective precursors for the synthesis of substituted thioureas and the construction of heterocyclic systems» (problem «Pharmacy», state registration No. 0118U004261, period of study 20172021). The work was performed with the financial support of «Enamine Ltd» (Kyiv, Ukraine).

\section{REFERENCES}

1. Aldenkamp AP, De Krom M, Reijs R. Newer antiepileptic drugs and cognitive issues. Epilepsia. 2003;44 Suppl 4:21-9.
2. Lundberg S, Eeg-Olofsson O. Rolandic epilepsy: a challenge in terminology and classification. Eur $J$ Paediatr Neurol. 2003;7(5):239-41.

3. Lima JM. The new drugs and the strategies to manage epilepsy. Curr Pharm Des. 2000;6(8):873-8.

4. Bialer $M$, White $H S$. Key factors in the discovery and development of new antiepileptic drugs. Nat Rev Drug Discov. 2010;9(1):68-82.

5. Al-Otaibi F. An overview of structurally diversified anticonvulsant agents. Acta Pharm. 2019;69(3):321-44.

6. Lee H, Le HV, Wu R, Doud E, Sanishvili R, Kellie JF, Compton PD, Pachaiyappan B, Liu D, Kelleher NL, Silverman $R B$. Mechanism of Inactivation of GABA Aminotransferase by (E)- and (Z)-(1S,3S)-3-Amino4-fluoromethylenyl-1-cyclopentanoic Acid. ACS Chem Biol. 2015;10(9):2087-98.

7. Masereel B, Lambert DM, Dogné JM, Poupaert JH, Delarge J. Anticonvulsant activity of pyrid-3-yl-sulfonyl ureas and thioureas. Epilepsia. 1997;38(3):334-7.

8. Pandeya SN, Mishra V, Singh PN, Rupainwar DC. Anticonvulsant activity of thioureido derivatives of acetophenone semicarbazone. Pharmacol Res. 1998;37(1):17-22.

9. Pandeya SN, Mishra V, Ponnilavarasan I, Stables JP. Anticonvulsant activity of p-chlorophenyl substituted arylsemicarbazones--the role of primary terminal amino group. Pol $J$ Pharmacol. 2000;52(4):283-90.

10. Celen AO, Kaymakcioglu B, Gumru S, Toklu HZ, Aricioglu F. Synthesis and anticonvulsant activity of substituted thiourea derivatives. Marmara Pharm J. 2011; 15: 43-7.

11. Kumar D, Sharma VK, Kumar R, Singh T, Singh H, Singh AD, Roy RK. Design, synthesis and anticonvulsant activity of some new 5,7-dibromoisatin semicarbazone derivatives. EXCLI J. 2013;12:628-40.

12. Celen AO, Kaymakcioglu B, Gumru S, Toklu HZ, Aricioglu $F$. Synthesis and anticonvulsant activity of substituted thiourea derivatives. Marmara Pharm J. 2011; 15: 43-7.

13. Aggarwal N, Mishra P. Synthesis of 4-aryl substituted semicarbazones of some terpenes as novel anticonvulsants. J Pharm Pharm Sci. 2004;7(2):260-4.

14. Divar M, Yeganeh Y, Jamshidzadeh A, Heidari R, Khabnadideh $S$. Anticonvulsant activity of some 
semicarbazone and thiosemicarbazone derivatives of isatin on PTZ induced seizure. J Innov Appl Pharm Sci. 2017; 2(3): 4-14.

15. Talele TT. The «Cyclopropyl Fragment» is a Versatile Player that Frequently Appears in Preclinical/Clinical Drug Molecules. J Med Chem. 2016;59(19):8712-56.

16. Agrawal S, Jain J, Kumar A, Gupta P, Garg V. Synthesis molecular modeling and anticonvulsant activity of some hydrazone, semicarbazone, and thiosemicarbazone derivatives of benzylidene camphor. Res Rep Med Chem. 2014; 4: 47-58.

17. Gajbhiye A. Synthesis and anticonvulsant activity of some 1-cyclohexylidine/ cycloheptylidine-4-substitued semicarbazide derivatives. Int J Pharmacol Pharmaceut Tech. 2017; 1(2): 51-6.

18. He X, Zhong M, Yang J, Wu Z, Xiao Y, Guo H, Hu X. Synthesis and anticonvulsant activity of 1-(2-(8-(benzyloxy)quinolin-2-yl)-1-butyrylcyclopropyl)-3-substituted urea derivatives. Chem Biol Drug Des. 2012;79(5):771-9.

19. Carruthers W, Coldham I. 'Modern Methods of Organic Synthesis 4th edition'. Cambridge: University Press, 2004; 493p.

20. Kholodniak OV, Kazunin MS, Meyer F, Kovalenko SI, Steffens KG. Novel N-CycloalkylcarbonylN'-arylthioureas: Synthesis, Design, Antifungal Activity and Gene Toxicity. Chem Biodivers. 2020;17(7):e2000212.

21. Macdonald RL, Greenfield LJ Jr. Mechanisms of action of new antiepileptic drugs. Curr Opin Neurol. 1997;10(2):121-8.

22. Hanaya R, Arita $K$. The New Antiepileptic Drugs: Their Neuropharmacology and Clinical Indications. Neurol Med Chir (Tokyo). 2016;56(5):205-20.

23. Trott $O$, Olson AJ. AutoDock Vina: improving the speed and accuracy of docking with a new scoring function, efficient optimization, and multithreading. J Comput Chem. 2010;31(2):455-61.

24. European convention for the protection of vertebrate animal used for experimental and other scientific purposes. Council of Europe: Strasbourg. 1986

25. Golovenko MA, Gromov LO. Doklinichne vivchennya spetsifichnoyi aktivnosti potentsiinih protisudomnih preparativ. Kyiv: Metodichni rekomendatsiyi. DFC MOZ Ukrainy, 2003; 26p/
26. Lapach SN, Chubenko AV, Babich PN. Statisticheskie metodyi v mediko-biologicheskih issledovaniyah s ispolzovaniem EXCEL. Kyiv: Morion, 2001, 408 p.

27. Breitmaier E. Structure Elucidation by NMR in Organic Chemistry. Wiley: A Practical Guide/Eberhard Breitmaier. 3rd Revised Edition, 2002; 68 p.

\section{Дизайн, синтез і протисудомна активність нових діацилтіосемікарбазидів}

О. В. Холодняк, В. В. Ставицький, М. С. Казунін, Н. В. Бухтіярова, Г. Г. Берест, І. Ф. Бєленічев, С. І. Коваленко

Мета. Спрямований пошук протисудомних агентів серед невідомих діацилтіосемікарбазидів з обговоренням взаємозв' язку «структура-активність». Методи. Органічний синтез; молекулярний докінг; спектральні методи; пентилентетразольні судоми, статистичні методи. Результати. Розроблена стратегія пошуку нових протисудомних агентів серед невідомих діацилтіосемікарбазидів $з$ використанням віртуально-орієнтованого скринінгу щодо активних центрів ферментів та натрієвих каналів, які лежать в основі механізму дії протиепілептичних препаратів. Синтез діацилтіосемікарбазидів проведено методом in situ, взаємодією циклоалканкарбонілхлоридів 3 ізотіоціанатом амонію 3 подальшим нуклеофільним приєднанням гідразидів циклоалкіл- (аралкіл-, арил-, гетарил-)карбонових кислот. Особливості будови синтезованих сполук підтверджені спектральними методами (хромато- та ${ }^{1} \mathrm{H}$-ЯМРспектри). Біологічний скринінг показав, що діацилтіосемикарбазиди (2) на експериментальній моделі пентилентеразольних судом у щурів збільшують латентний період судом у 2.77-7.82 рази, зменшують тривалості тоніко-клонічних нападів на $1.23-5.59$ хв. та запобігають смертності на 30-60 \%, відносно до контрольної групи тварин. Показано, що діацилтіосемікарбазиди $(2.6,2.15,2.22,2.18)$ з циклопропан(циклопентан-)карбоксамідними групами виявляють протисудомну активність, що перевищує або конкурує з еталонним препаратом Депакін. Висновки. Отримано ряд нових діацилтіосемікарбазидів, проведено первинний скриніг на протисудомну активність, обговорено взаємозв'язок «структура-активність» та виявлена 
активна сполука для подальших більш глибоких фармакологічних досліджень.

Кл ю ч о в і с л о в а: діацилтіосемікарбазиди, дизайн, синтез, пентилентетразольні судоми, протисудомна активність.

\section{Дизайн, синтез и противосудорожное активность новых диацилтиосемикарбазидов}

Е. В. Холодняк, В. В. Ставицкий, М. С. Казунин, Н. В. Бухтиярова, Г. Г. Берест, И. Ф. Беленичев, С. И. Коваленко

Цель. Направленный поиск противосудорожных агентов среди неизвестных диацилтиосемикарбазидов с обсуждением взаимосвязи «структура-активность». Методы. Органический синтез; молекулярный докинг; спектральные методы; пентилентетразольные судороги, статистические методы. Результаты. Разработана стратегия поиска новых противосудорожных агентов среди неизвестных диацилтиосемикарбазидов с использованием виртуально-ориентированного скрининга к активным центрам ферментов и натриевых каналов, лежащих в основе механизма действия противоэпилептических препаратов. Синтез диацилтиосемикарбазидов проведено методом in situ, взаимодействием циклоалканкарбонилхлоридов с изотиоцианатом аммония с последующим нуклеофильным присоеди- нением гидразидов циклоалкил-(аралкил-, арил, гетарил-)карбоновых кислот. Особенности строения синтезированных соединений подтверждены спектраль-

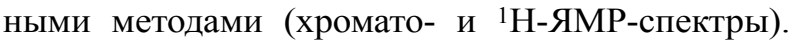
Биологический скрининг показал, что диацилтиосемикарбазиды (2) на экспериментальной модели пентилентеразольних судорог у крыс увеличивают латентный период судорог в 2.77-7.82 раза, уменьшают продолжительность тонико-клонических приступов на 1.23-5.59 мин. и предотвращают смертность на 30$60 \%$, по отношению к контрольной группе животных. Показано, что диацилтиосемикарбазиды $(2.6,2.15$, $2.22,2.18)$ с циклопропан-(циклопентан-) карбоксамидной группой у молекуле проявляют противосудорожную активность, превышающую или конкурирующую с эталонным препаратом Депакин. Выводы. Получен ряд новых диацилтиосемикарбазидов, проведен первичный скриниг на противосудорожную активность, обсуждена взаимосвязь «структура-активность» и выявлено активное соединение для дальнейших более глубоких фармакологических исследований.

К л юч е в ы е с л ов а: диацилтиосемикарбазиды, дизайн, синтез, пентилентетразольные судороги, противосудорожная активность.

Received 11.12.2020 